\title{
Modeling of non-equilibrium effects in intermittency region between two phases
}

\author{
Tomasz Wacławczyk,* \\ Warsaw University of Technology, Institute of Aeronautics and Applied Mechanics, \\ Division of Aerodynamics \\ ul. Nowowiejska 24, 00653 Warszawa, Poland
}

\begin{abstract}
This paper concerns modeling of the evolution of the intermittency region between two weakly miscible phases due to temporal and spatial variations of its characteristic length scale. First, the need for a more general description allowing for the evolution of the intermittency region is rationalized. Afterwards, results of the previous work (Wacławczyk T., 2017, On a relation between the volume of fluid, level-set and phase field interface models, Int. J. Multiphas. Flow, Vol. 97) are discussed in the context of sharp interface models known in the literature and new insight into droplet coalescence mechanism recently recognized in molecular dynamics studies (Perumanath S., Borg M.K., Chubynsky M.V., Sprittles J.E., Reese J.M., 2019, Droplet coalescence is initiated by thermal motion, Phys. Rev. Lett., Vol. 122). Finally, physical and numerical models extending the applicability of the equilibrium solution to the case when the intermittency region could also be in the non-equilibrium state are introduced and verified.
\end{abstract}

Keywords: non-equilibrium diffusive interface model, intermittency region evolution, variable characteristic length-scale, turbulent two-phase flow

\footnotetext{
*Tomasz Wacławczyk

Email address: tomasz.waclawczyk@pw.edu.pl (Tomasz Wacławczyk)
} 


\section{Introduction}

The gas-liquid interface is a domain where material properties of two adjacent phases are changing. However, "the exact definition of the gas-liquid interface is nebulous" (Faust, 2018). In fluid dynamics, there are two accepted physical models of the gas-liquid interface, namely: the dividing surface model (Gibbs, 1874) and the diffusive interface model (van der Waals, 1893). In a recent review paper (Elghobashi, 2019) concerning direct numerical simulation (DNS) of the turbulent, dispersed two-phase flows, numerical methods inspired by these two physical models of the gas-liquid interface are listed as "the tracking scalar approach". The dividing surface model of Gibbs is the foundation of the volume of fluid (VOF) (Tryggvason et al., 2011; Lu and Tryggvason, 2018) and standard level-set (SLS) (Osher and Sethian, 1988; Osher and Fedkiw, 2003; Sussman et al., 2007; Deike et al., 2016) sharp interface methods. The diffusive interface model of van der Waals stimulated development of the phase-field methods based on the Cahn-Hilliard (Cahn and Hilliard, 1958; Anderson et al., 1998; Komrakova et al., 2015; Fedeli, 2017; Soligo et al., 2019) and modified Allen-Cahn (Allen and Cahn, 1979; Olsson and Kreiss, 2005; Chiu and Lin, 2011; McCaslin and Desjardins, 2014; Wacławczyk, 2015; Gruszczyński et al., 2020; Kajzer and Pozorski, 2020; Mirjalili et al., 2020) equations.

A common feature of the aforementioned physical and numerical models is the assumption that the gas-liquid interface is a boundary (geometric object) evolving in the given (turbulent) velocity field. Consequently, in accordance with the postulates of the DNS, the velocity field has to be resolved to the Kolmogrov

length scale $\sim R e^{-3 / 4}$ to reconstruct all time and length scales governing its evolution. However, millions of droplets or bubbles created in the effect of violent topological changes can easily have the diameter below the Kolmogrov length scale (Elghobashi, 2019). To model their sub-grid dynamics and its impact on the resolved flow field, phenomenological models are used.

To increase the range of Reynolds numbers where numerical simulations can offer useful predictions, some reduced models are obtained in the course of fil- 
tering or ensemble averaging of the two-phase flow governing equations. These operations result, respectively, in the large-eddy (LES) (Labourasse et al., 2007; Toutant et al., 2007; Aniszewski et al., 2012; Herrmann, 2013; Saeedipour and Schneiderbauer, 2019) and Reynolds averaged (RANS) (Hong and Walker, 2000; Guo and Shen, 2010) formulations of the one-fluid model. After filtering or ensemble averaging of one-fluid model equations, phenomenological models (often based on the DNS) are used to close correlations between the instantaneous (sub-grid) macroscopic interface and turbulent-velocity field (sub-grid) fluctuations. Regardless of the reduction in the number of degrees of freedom, the gas-liquid interface in the filtered/averaged one-fluid model is approximated in the same way as in the DNS. The characteristic scalar function defining the gasliquid interface is transported using filtered or ensemble averaged fluid velocity. Hence, the gas-liquid interface is once again viewed as the passive boundary between gas-liquid phases. As a consequence, the gas-liquid interface model in the DNS, LES or RANS formulations of the one-fluid model does not play an active role in the modeling process. In addition the aforementioned phenomenological models are often based on a different modeling strategy than the one(s) used in the one-fluid model, e.g. two-fluid or Euler-Lagrange frameworks (Prosperetti and Tryggvason, 2007; Elghobashi, 2019). This introduces coupling and feedback problems that must be addressed during time-consuming simulations.

Recently, the present author (Wacławczyk, 2017) has shown the mathematical models describing the gas-liquid interface listed by (Elghobashi, 2019) as "the tracking scalar approach" are complementary components of the gas-liquid interface statistical description. Herein, this result is extended and used to propose modeling framework that is natural for the one-fluid model of (turbulent) two-phase flow.

In the present work it is assumed that the macroscopic intermittency region is a domain where the gas-liquid interface $\Gamma$ can be found with non-zero probability. This description was first introduced for the modeling of turbulence/gasliquid interface interactions (Brocchini and Peregrine, 2001a,b). Therein, the sharp interface $\Gamma$ is the gas-liquid interface, its ensemble averaged oscillations 
create the macroscopic intermittency region evolving due to the stochastic, unsteady nature of the turbulent flow. In this interpretation, deformations of the sharp interface $\Gamma$ are caused by stochastic forcing of the turbulent eddies described typically in terms of the characteristic time and length scales. These characteristic scales are altered by gravity and surface tension forces. The phenomenological model of Brocchini and Peregrine was used by several authors (Hong and Walker, 2000; Smolentsev and Miraghaie, 2005; Höhne and Vallée, 2009; Wacławczyk and Oberlack, 2011; Skartlien et al., 2014) to propose quantitative models of turbulence/gas-liquid interface interactions. In particular, Wacławczyk and Oberlack have proposed the correlation between the local interface $\Gamma$ position and velocity fluctuation in the normal direction $\mathbf{n}_{\Gamma}$ must be modeled to account for the evolution of the intermittency region. This idea was used to analyze the intermittency region evolution based on a priori study of turbulent velocity field in the vicinity of the sharp interface (Wacławczyk et al., 2014; Wacławczyk and Wacławczyk, 2015). Therein it was found that the intermittency region characteristic time and length scales are not constant but can vary in time and space.

Next, the present author (Wacławczyk, 2017) has shown that the intermittency region paradigm can be used to derive the equilibrium condition for the non-flat, gas-liquid interface $\gamma$ (mesoscopic intermittency region). The main argument therein is based on the analogy between processes of turbulence/gasliquid interface and thermal-fluctuations/mesoscopic interface interactions. As argued by (Brocchini and Peregrine, 2001a,b) and recently confirmed in molecular dynamics studies by (Perumanath et al., 2019) both processes are stochastic in their nature. This means the derivation of the macroscopic (averaged) equations governing their evolution requires conditional averaging taking into account the instantaneous position of the sharp interface $\Gamma$. Moreover, one expects that in the limit of vanishing energy of turbulent or thermal fluctuations, a more general, statistical model of the intermittency region should be reduced to sharp interface models known in the literature.

In the present work, the analogy between turbulence/gas-liquid interface 


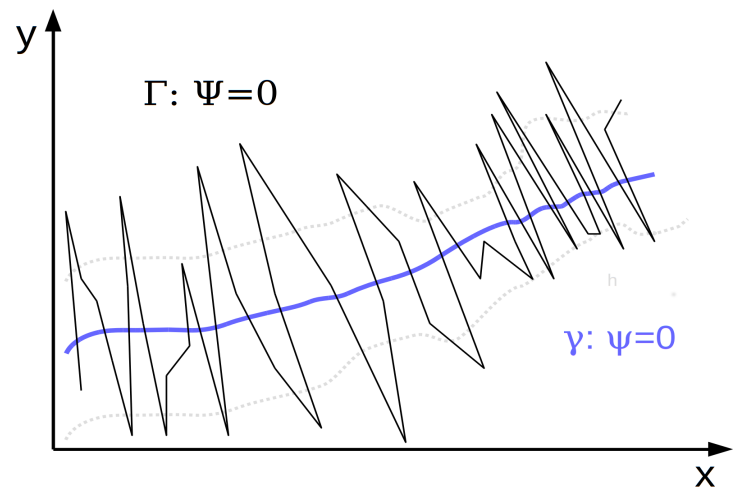

Figure 1: Sketch of one realization of the considered stochastic process, the sharp interface $\Gamma: \Psi(\mathbf{x}, t)=0$ (black-solid line) disturbed by the field of stochastic forces is oscillating around its expected position $\gamma: \psi(\mathbf{x}, t)=0$ (blue-solid line) that defines the regularized interface. The intensity of $\Gamma: \Psi(\mathbf{x}, t)=0$ oscillations can in general be variable in space and/or time.

and thermal-fluctuations/mesoscopic interface interactions is further exploited. We note, when the gas-liquid interface is in the equilibrium state, the characteristic length scale $\epsilon_{h}(\mathbf{x}, t)[m]$ governing its thickness is constant in time and space. In the converse case the gas-liquid interface is in the non-equilibrium state. This scheme is extended to the case of turbulence/gas-liquid interface interactions. The distinction between equilibrium and non-equilibrium states of the mesoscopic intermittency region, allows the assessment of the extent to which classical sharp/diffusive interface models account for the stochastic characteristics of the gas-liquid interface and explains why they allow predictions of topological changes governed by the molecular effects (Perumanath et al., 2019). Further in the paper, the physical models and numerical methods which allow $\epsilon_{h}(\mathbf{x}, t)$ to be variable in time and space are proposed and used during numerical solution of the intermittency region evolution equation.

The present paper is organized as follows. In Section 2, the equation governing the evolution of the intermittency region is derived from the stochastic viewpoint and the conditions for the intermittency region equilibrium and nonequilibrium are elucidated. Afterwards, it is argued why the sharp/diffusive 
models of the intermittency region have the potential to predict topological changes during break up or coalescence occurring on a molecular level. In Section 3 it is shown that the stationary solution of the intermittency region evolution equation accounting for variable $\epsilon_{h}(\mathbf{x}, t)$ is equivalent to the minimization of the corresponding energy functional. This result permits the stationary solution of the intermittency region evolution equation to be used as the local equilibrium condition. In Section 3.2, the local equilibrium condition accounting for $\epsilon_{h}(\mathbf{x}, t)$ is employed to derive the mapping function that accounts for the non-equilibrium effects. Next, the generalized mapping function is used during numerical solution of the intermittency region evolution equation. In Section 4, the numerical method employed to integrate the intermittency region evolution equation is described and results of the intermittency region evolving due to $\epsilon_{h}(\mathbf{x}, t)$ are presented and discussed. Section 5 provides the conclusions and perspectives for future work.

\section{Derivation of the intermittency region evolution equation}

Let us assume the evolution of mesoscopic sharp interface $\Gamma$ is governed by the phase indicator function $H_{\Gamma}(\Psi(\mathbf{x}, t))$ transport equation

$$
\frac{\partial H_{\Gamma}}{\partial t}+\mathbf{W} \cdot \nabla H_{\Gamma}=\frac{\partial H_{\Gamma}}{\partial t}+\delta_{\Gamma}(\Psi)|\nabla \Psi| \mathbf{W} \cdot \mathbf{n}_{\Gamma}=0,
$$

where $\Psi(\mathbf{x}, t)[m]$ is the signed distance function from the points $\delta_{\Gamma}(\Psi)[1 / m]$ located on the two-dimensional surface $\Psi(\mathbf{x}, t)=0$ defining the sharp interface $\Gamma$ with the normal vector $\mathbf{n}_{\Gamma}=\nabla \Psi /|\nabla \Psi|$, see solid-black line in Fig. 1. $\mathbf{W} \cdot \mathbf{n}_{\Gamma}[\mathrm{m} / \mathrm{s}]$ is the stochastic velocity field governing the motion of $\delta_{\Gamma}(\Psi) . H_{\Gamma}(\Psi(\mathbf{x}, t))$ represents one, instantaneous realization of the stochastic process generated by thermal fluctuations $\mathbf{W}^{\prime}=\mathbf{W}-\langle\mathbf{W}\rangle[\mathrm{m} / \mathrm{s}]$. For this reason, Eq. (1) is of no use in the continuous description of gas/fluid systems. To derive its continuum version the ensemble averaging (Pope, 1998; Wacławczyk and Oberlack, 2011; Wacławczyk, 2017) must be applied to Eq. (1). It is noticed, unlike in the recent work (Thiesset et al., 2020), analysis in the present paper is based on one-point surface statistics. 
The ensemble average of the first LHS term in Eq. (1) results in

$$
\frac{\partial}{\partial t}\left\langle H_{\Gamma}(\Psi)\right\rangle=\frac{\partial}{\partial t} \int_{-\infty}^{\infty} H_{\Gamma}(\xi)\langle\delta(\Psi(\mathbf{x}, t)-\xi)\rangle d \xi
$$

where $\langle\delta(\Psi(\mathbf{x}, t)-\xi)\rangle$ is the ensemble average $\langle\cdot\rangle$ of the fine grained p.d.f.'s $\delta(\Psi(\mathbf{x}, t)-\xi)$ characterizing each realization of the stochastic process in the sample space $\xi[m] .\langle\delta(\Psi(\mathbf{x}, t)-\xi)\rangle$ provides the probability density that $\xi<$ $\Psi(\mathbf{x}, t)<\xi+d \xi$.

The contributions to the ensemble average $\langle\cdot\rangle$ from the second RHS term in Eq. (1) are non-zero only if the interface $\Gamma$ is present at point $\mathbf{x}$ and time $t$ where/when the averaging is carried out. This issue was recognized by (Pope, 1998) who proposed the conditional surface average $\langle\cdot\rangle_{\Gamma}$ to account for the smearing of the interface $\Gamma$ due to the averaging process. Its application to the second RHS term in Eq. (1) leads to

$$
\left\langle\mathbf{W} \cdot \nabla H_{\Gamma}\right\rangle=\left\langle\mathbf{W} \cdot \mathbf{n}_{\Gamma} \delta_{\Gamma}(\Psi)\right\rangle=\left\langle\mathbf{W} \cdot \mathbf{n}_{\Gamma}\right\rangle_{\Gamma} \Sigma
$$

where $\langle\cdot\rangle_{\Gamma}$ is the surface average

$$
\left\langle\mathbf{W} \cdot \mathbf{n}_{\Gamma}\right\rangle_{\Gamma}=\frac{1}{\Sigma} \iint_{\Gamma}\left\langle\mathbf{W} \cdot \mathbf{n}_{\Gamma} \delta(\mu) \delta(\lambda) \delta\left(\Psi-\Psi^{\prime}(\mu, \lambda, t)\right) A(\mu, \lambda, t)\right\rangle d \mu d \lambda
$$

and $\Sigma[1 / m]$ is given by the formula

$$
\Sigma=\iint_{\Gamma}\left\langle\delta(\mu) \delta(\lambda) \delta\left(\Psi-\Psi^{\prime}(\mu, \lambda, t)\right) A(\mu, \lambda, t)\right\rangle d \mu d \lambda .
$$

In equations (4-5) $\mu, \lambda, \Psi$ define the local, orthonormal coordinate system of the infinitesimally small element $A(\mu, \lambda, t) d \mu d \lambda$ where $\Psi$ is the coordinate in the normal direction. $\Sigma[1 / m]$ can be interpreted as the amount of the expected surface-to-volume ratio (Pope, 1998); in the general case $\Sigma(\mathbf{x}, t)$ in Eq. (3) is unknown and must be closed by a model. Using the decomposition $\mathbf{W}=$ $\langle\mathbf{W}\rangle+\mathbf{W}^{\prime}$ and Eqs. (3-5) one obtains

$$
\left\langle\mathbf{W} \cdot \mathbf{n}_{\Gamma}\right\rangle_{\Gamma} \Sigma=\langle\mathbf{W}\rangle\left\langle\mathbf{n}_{\Gamma}\right\rangle_{\Gamma} \Sigma+\left\langle\mathbf{W}^{\prime} \cdot \mathbf{n}_{\Gamma}\right\rangle_{\Gamma} \Sigma
$$

Next, the exact relations $\left\langle\mathbf{W}^{\prime} \cdot \nabla H_{\Gamma}\right\rangle=\left\langle\mathbf{W}^{\prime} \cdot \mathbf{n}_{\Gamma}\right\rangle_{\Gamma} \Sigma$ and $\left\langle\mathbf{n}_{\Gamma}\right\rangle_{\Gamma} \Sigma=\nabla\left\langle H_{\Gamma}\right\rangle$, see Eqs. (A.1) and (A.8) respectively, lead to averaged Eq. (1) with the RHS term 
that must be closed

$$
\frac{\partial \alpha}{\partial t}+\mathbf{w} \nabla \alpha=-\left\langle\mathbf{W}^{\prime} \cdot \mathbf{n}_{\Gamma}\right\rangle_{\Gamma} \Sigma=-\left\langle\mathbf{W}^{\prime} \cdot \mathbf{n}_{\Gamma} \delta_{\Gamma}(\Psi)\right\rangle=-\left\langle\mathbf{W}^{\prime} \cdot \nabla H_{\Gamma}\right\rangle,
$$

where we have denoted $\alpha=\left\langle H_{\Gamma}\right\rangle$ and $\mathbf{w}=\langle\mathbf{W}\rangle$.

As it was put forward by (Wacławczyk and Oberlack, 2011), the unknown RHS term in Eq. (7) can be closed by the eddy diffusivity model

$$
\left\langle\mathbf{W}^{\prime} \cdot \mathbf{n}_{\Gamma}\right\rangle_{\Gamma} \Sigma=-D \nabla \cdot\left\langle\mathbf{n}_{\Gamma}\right\rangle_{\Gamma} \Sigma
$$

Taking the divergence of the exact relation $\left\langle\mathbf{n}_{\Gamma}\right\rangle_{\Gamma} \Sigma=\nabla \alpha$ leads to

$$
\nabla \cdot\left\langle\mathbf{n}_{\Gamma}\right\rangle_{\Gamma} \Sigma=\nabla^{2} \alpha-\left\langle\mathbf{n}_{\Gamma}\right\rangle_{\Gamma} \cdot \nabla \Sigma
$$

Substitution of Eq. (9) and Eq. (8) into Eq. (7) results in

$$
\frac{\partial \alpha}{\partial t}+\mathbf{w} \nabla \alpha=D \nabla^{2} \alpha-D\left\langle\mathbf{n}_{\Gamma}\right\rangle_{\Gamma} \cdot \nabla \Sigma
$$

where the second, unclosed RHS term in Eq. (10) was identified by Wacławczyk and Oberlack as counter gradient diffusion. The above equation is not in the desired conservative form. Thus, with the help of exact relation $\left\langle\mathbf{n}_{\Gamma}\right\rangle_{\Gamma} \Sigma=\nabla \alpha$, taking into account the case when $D(\mathbf{x}, t)=C \epsilon_{h}(\mathbf{x}, t)$ and noting the vector normal to the regularized interface $\mathbf{n}_{\gamma}=\nabla \alpha /|\nabla \alpha|=\left\langle\mathbf{n}_{\Gamma}\right\rangle_{\Gamma} /\left|\left\langle\mathbf{n}_{\Gamma}\right\rangle_{\Gamma}\right|$, Eq. (10) is rewritten as

$$
\frac{\partial \alpha}{\partial t}+\mathbf{w} \nabla \alpha=\nabla \cdot(D \nabla \alpha)-\left|\left\langle\mathbf{n}_{\Gamma}\right\rangle_{\Gamma}\right| \nabla(D \Sigma) \cdot \mathbf{n}_{\gamma} .
$$

One notes that Eq. (11) accounts for the variable characteristic length scale $\epsilon_{h}(\mathbf{x}, t)$, however, it is still unclosed due to the presence of the counter gradient diffusion term. The conservative closure of this unknown term (Wacławczyk and Wacławczyk, 2015) leads to the equation introduced by (Chiu and Lin, 2011) inspired by the conservative level-set (CLS) method (Olsson and Kreiss, 2005)

$$
\frac{\partial \alpha}{\partial t}+\nabla \cdot(\mathbf{w} \alpha)=\nabla \cdot\left[D|\nabla \alpha| \mathbf{n}_{\gamma}-C \alpha(1-\alpha) \mathbf{n}_{\gamma}\right]
$$

where $\mathbf{w}[\mathrm{m} / \mathrm{s}]$ is velocity of the regularized interface $\gamma$, and in the general case $C(\mathbf{x}, t)[\mathrm{m} / \mathrm{s}]$ and $D=C \epsilon_{h}(\mathbf{x}, t)\left[\mathrm{m}^{2} / \mathrm{s}\right]$ are velocity and diffusivity scales characterizing the intermittency region, respectively. The coefficients $C, D$ uniquely 
specify the characteristic length $\epsilon_{h} \sim D / C[m]$ and time $\tau_{h} \sim \epsilon_{h} / C[s]$ scales governing the solution of Eq. (12). We note the presence of the two RHS terms in Eq. (12) is supported by the fact that forces always occur in pairs. In Eq. (12) due to the presence of contraction $C \alpha(1-\alpha) \mathbf{n}_{\gamma}$, diffusion $D|\nabla \alpha| \mathbf{n}_{\gamma}$ is counterbalanced. The former term was identified as the first order approximation of joint probability of creation of the bond between particles of two different types (Cahn and Hilliard, 1958).

The steady state solution of Eq. (12) with $\epsilon_{h}=$ const. and $\mathbf{w}=\mathbf{u}=0$ is given by the regularized Heaviside function

$$
\alpha(\psi)=\frac{1}{1+\exp \left(-\psi(\mathbf{x}, t) / \epsilon_{h}\right)}=\frac{1}{2}\left[1+\tanh \left(\frac{\psi(\mathbf{x}, t)}{2 \epsilon_{h}}\right)\right]
$$

and its inverse function that is the signed distance from the expected position of the regularized interface $\gamma$ defined by the level-set $\psi(\alpha=1 / 2)=0$

$$
\psi(\alpha)=\epsilon_{h} \ln \left[\frac{\alpha(\psi)}{1-\alpha(\psi)}\right] .
$$

As noticed by the present author (Wacławczyk, 2015), Eqs. (13) and (14) are known to characterize the cumulative distribution $\alpha(\psi)$, and quantile $\psi(\alpha)$ functions of the logistic distribution. Additionally, the gradient of $\alpha(\psi)$ given by the formula

$$
\nabla \alpha=\frac{\tilde{\delta}(\alpha)}{\epsilon_{h}} \nabla \psi
$$

where $\tilde{\delta}(\alpha) / \epsilon_{h}=\alpha(1-\alpha) / \epsilon_{h}$ is the probability density function of the logistic distribution. Substitution of Eq. (15) into Eq. (12) gives

$$
\frac{\partial \alpha}{\partial t}+\mathbf{w} \nabla \alpha=\nabla \cdot\left[C \tilde{\delta}(\alpha)(|\nabla \psi|-1) \mathbf{n}_{\gamma}\right]
$$

where $\mathbf{n}_{\gamma}=\nabla \alpha /|\nabla \alpha|=\nabla \psi /|\nabla \psi|$, and $\mathbf{w}$ in Eq. (14) may now be replaced by the fluid velocity $\mathbf{u}$ since in absence of phase changes and/or advection $\mathbf{n}_{\gamma} \cdot \mathbf{w}=0$.

In the present work we separate the advection and re-initialization steps in Eq. (16), which leads to

$$
\frac{\partial \alpha}{\partial t}+\mathbf{w} \nabla \alpha=\frac{\partial \alpha}{\partial t}+\frac{\tilde{\delta}(\alpha)}{\epsilon_{h}} \mathbf{w} \cdot \nabla \psi=0,
$$




$$
\frac{\partial \alpha}{\partial \tau}=\nabla \cdot\left[C \tilde{\delta}(\alpha)(|\nabla \psi|-1) \mathbf{n}_{\gamma}\right] .
$$

This form of Eq. (12) is preferred. It allows to consider separately advection equation Eq. (17) and the model of the evolution of the intermittency region using Eq. (18). Solution of Eq. (18), known in the literature as the re-initialization step, was shown to be equivalent to minimization of the interfacial energy functional containing the term which accounts for the regularized interface $\gamma$ deformation. For this reason, it was argued in the previous work (Wacławczyk, 2017) that Eqs. (17) and (18) with the mapping between $\alpha(\psi)-\psi(\alpha)$ functions given by Eq. (14) describe the non-flat, intermittency region in the equilibrium state as therein $C=$ const., $D=C \epsilon_{h}=$ const.. Furthermore, the statistical interpretation of Eqs. (17) and (18) based on Eqs. (13)-(15) reveals the relation between the sharp and diffusive interface models.

As it was mentioned in Section 1, Eq. (12) can be interpreted as the statistical model of the mesoscopic or macroscopic intermittency region. The physical interpretation depends upon the character of the stochastic force field inducing W in Eq. (1) and chosen time/length scales. In the mesoscopic interpretation, the deformation of the sharp interface $\Gamma$ is caused by random, thermal fluctuations. In the macroscopic interpretation, velocity $\mathbf{W}$ in Eq. (1) can be related to the instantaneous turbulent velocity field. In the next section, terms under which the intermittency region is in the equilibrium or non-equilibrium state are discussed.

\subsection{Equilibrium and non-equilibrium state of the intermittency region}

First, the mesoscopic interface $\Gamma$ agitated by the thermal fluctuations is considered, see Fig. 1. After conditional averaging described in Section 2 its evolution is described by Eq. (12). Let us note $\alpha(\psi)$ and $\tilde{\delta}(\alpha)$ in Eq. (12) have infinite support as $\epsilon_{h} \sim \sqrt{k_{B} T / \sigma}>0$ where $k_{B}[J / K]$ is the Boltzman constant, $T[K]$ is the absolute temperature and $\sigma\left[\mathrm{J} / \mathrm{m}^{2}\right]$ is the surface tension coefficient (Vrij, 1973; Aarts et al., 2004). $k_{B} T / V\left[\mathrm{~J} / \mathrm{m}^{3}\right]$ is the root mean square measure of the thermal fluctuations of molecules acting to distort the mesoscopic interface 
$\Gamma$ between two phases in the infinitesimally small volume $V$. The amount of thermal energy in $V$ determines the kinetic energy of molecules. A surface tension $\sigma / V\left[\mathrm{~J} / \mathrm{m}^{5}\right]$ represents net work done by the cohesive forces between fluid molecules per unit area of $\Gamma$ in $V$. The cohesive forces between fluid molecules act to suppress increases in interfacial area of $\Gamma$.

In the case of the turbulence/gas-liquid interface interactions, the interface $\Gamma$ in Fig. 1 is the sharp representation of the gas-liquid, macroscopic interface. By analogy to the mesoscopic case, the characteristic length scale $\epsilon_{h}(\mathbf{x}, t)$ is governed by the ratio of net turbulent kinetic energy $\rho k\left[\mathrm{~J} / \mathrm{m}^{3}\right]$ and the work of forces generating turbulent stresses per unit area of the interface $\Gamma$ in $V,\left[\mathrm{~J} / \mathrm{m}^{5}\right]$. These forces are acting to decrease or increase the sharp interface $\Gamma$ interfacial area. In the macroscopic interpretation of Eq. (12), the ratio of work done by volume/surface forces in the intermittency region is altered by the work done by the gravitational and surface tension forces, respectively.

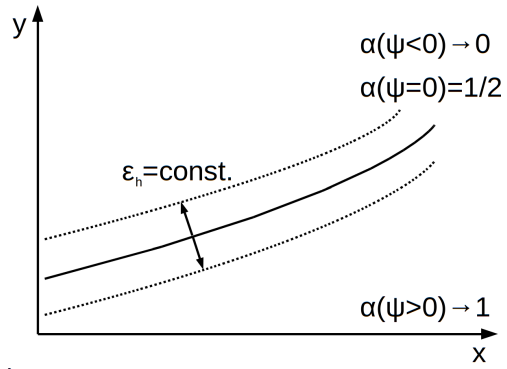

a)

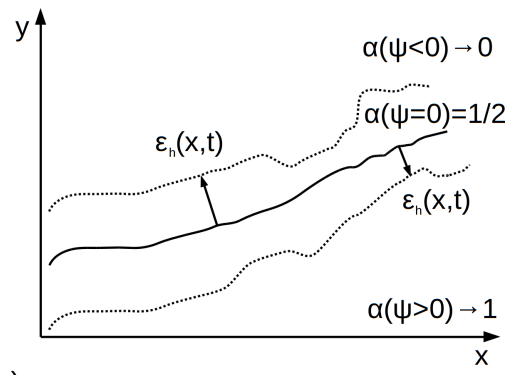

b)

Figure 2: Schematic picture of the intermittency region in (a) equilibrium $\epsilon_{h}=$ const. and (b) non-equilibrium $\epsilon_{h}(\mathbf{x}, t)$ states. The expected position of the interface $\gamma$ is marked with a black-solid line.

If the ratio of work done by the random volume forces (inducing fluctuations of $\Gamma$ ) and surface forces (per unit area of $\Gamma$ ) is constant in time and space, then also $\epsilon_{h}(\mathbf{x}, t)=$ const. and the intermittency region is in the equilibrium state, see Fig. 2a. In the opposite case, the characteristic length scale $\epsilon_{h}(\mathbf{x}, t)$ may change in time and space, and for this reason, the intermittency region is in the non-equilibrium state, see Fig. 2b. 
In what follows, the statistical interpretation of Eq. (12) is used to localize the sharp interface tracked or captured in the VOF, SLS sharp interface models.

\subsection{Stochastic coalescence and sharp/diffusive interface models}

In a series of molecular dynamics simulations of two droplets collisions (Perumanath et al., 2019) have identified the characteristic thermal length scale $l_{T} \sim 2 \sqrt{\epsilon_{h} R}$ where $\epsilon_{h} \sim \sqrt{k_{B} T / \sigma}[m]$ is the intermittency region thickness, $R[m]$ denotes colliding droplets radii. The existence of the thermal length scale $l_{T}[\mathrm{~m}]$ shows that before the capillary forces take control on the droplets coalescence, molecular and thermal effects govern this process. Therefore, Perumanath et al. conclude that droplet coalescence is a stochastic phenomenon initiated by thermal motion of fluid particles. Since $l_{T}[m]$ is proportional to the square root of the droplet radius $R[m]$, it is expected that during topological changes the molecular effects may influence phenomena on the macroscopic scale resolved in fluid dynamics.

In light of this fact, classical sharp interface models seem to overlook molecular effects (see derivations the signed-distance and phase indicator function transport equations in (Osher and Fedkiw, 2003; Tryggvason et al., 2011), respectively. This gives rise to the question of the extent to which the sharp interface models are able to reconstruct topological changes of the gas-liquid interface during coalescence and/or break up events.

First we note the expected position of the regularized (macroscopic) interface $\gamma: \psi=0$ is different than the instantaneous position of the mesoscopic sharp interface $\Gamma: \Psi=0$ defining one realization of the stochastic process, see Fig. 1. Since in the case of gas-liquid intermittency region in the equilibrium state $0<\epsilon_{h} \ll 1$ and $\epsilon_{h}=$ const., one can assume $\epsilon_{h}$ does not depend on volume and surfaces forces doing work in the domain where a two-phase system is changing its properties. Hence, $\epsilon_{h} \rightarrow 0$ means $\alpha(\psi) \rightarrow H_{\gamma}(\psi=0)=1 / 2$. For this reason, similar to the Gibbs dividing surface neglecting information about $0<\epsilon_{h} \ll 1$, sharp interface models are valid only when it is assumed that the intermittency region is in the equilibrium state. 
The phase indicator function $H_{\gamma}$ built on the expected position of the regularized interface $\gamma: \psi(\mathbf{x}, t)=0$ is different than the phase indicator function $H_{\Gamma}$ built on the signed distance function $\Psi(\mathbf{x}, t)$, as $H_{\Gamma}(\Psi(\mathbf{x}, t))$ is one realization of the stochastic process governed by Eq. (1). $H_{\gamma}$ is the phase indicator function discretized in VOF methods, $\psi(\mathbf{x}, t)$ is the signed distance function discretized in SLS methods. The level-sets $H_{\gamma}=1 / 2, \psi=0$ are two equivalent geometric, two-dimensional representations of the expected position of the gasliquid regularized interface $\gamma$ as $0<\epsilon_{h} \ll 1$ must remain greater than zero. This explains how the molecular effects are taken into account in VOF, SLS type sharp interface models.

Because the sharp interface approximations are formulated in the limit of $\epsilon_{h} \rightarrow 0$, they do not depend explicitly on $\epsilon_{h}$. Hence, they do not allow to account for the non-zero volume/interfacial energy ratio governing the intermittency region width, its possible variations and consequences of these, too. Thus, without additional modeling assumptions VOF, SLS type sharp interface models can not account for thermal effects described by (Perumanath et al., 2019). However, due to the fact VOF, SLS interface models sharply reconstruct the expected position of the regularized gas-liquid interface $\gamma: H_{\gamma}=1 / 2, \psi=0$, respectively, they are able to approximate break up and (in most of the cases) coalescence processes.

As long as the intermittency region remains in the equilibrium state and/or energy of stochastic fluctuations is small and independent of background physical phenomena the sharp interface model is a good approximation. In the opposite case, some physical effects may be lost when using the sharp interface model as local variations of the volume/surface forces work ratio in the intermittency region can affect dynamics of adjacent gas-liquid phases, for example through the local modifications of their material properties. The remaining part of the present paper proposes how the description given by Eqs. $(17,18,14)$ can be extended to model the intermittency region in the non-equilibrium state. 


\section{Modeling of non-equilibrium effects in the intermittency region}

As it has been explained in the previous sections, the motivation for generalized numerical solution of Eqs. $(17,18,14)$ comes from the need to account for the case when the ratio of work done by the volume/surface forces governing $\epsilon_{h}$ varies in space and time. Generalization of the equilibrium model can also be justified from a thermodynamic perspective. The intermittency region between two weakly miscible phases is an open system that may not be in the equilibrium state as it is perpetually exchanging energy with neighboring phases.

During previous analytical considerations and numerical experiments it has been assumed that $C=$ const. and $\epsilon_{h} \sim \Delta x$, see Fig. 2a. The main subject of the present section is to extend the analytical model and numerical solution of the set of partial differential algebraic Eqs. $(17,18,14)$ to the case when $\epsilon_{h}(\mathbf{x}, t)$ is variable as it is schematically depicted in Fig. $2 \mathrm{~b}$. The case when the characteristic time scale $\tau_{h} \sim \epsilon_{h}(\mathbf{x}, t) / C(\mathbf{x}, t)$ is variable as well is left for future studies.

\subsection{Minimization of free energy functional with variable characteristic length} scale

In the previous work of the present author (Wacławczyk, 2017) it has been shown the Helmholtz free energy functional defining the energy of the two-phase system

$$
F[\alpha]=\int_{V} \sigma\left[\epsilon_{h}|\nabla \alpha|^{2}+\frac{f(\alpha)}{\epsilon_{h}}+k(\alpha)\right] d V
$$

where $\sigma\left[\mathrm{J} / \mathrm{m}^{2}\right]$ is a known constant and $f(\alpha)=\alpha^{2}(1-\alpha)^{2}[-]$, has to contain term $k(\alpha)$ accounting for the energy of the regularized interface $\gamma$ deformation. Its presence in Eq. (19) is required to guarantee the equilibrium state of the nonflat regularized interface $\gamma$ by setting $\delta F / \delta \alpha=0$. From the equilibrium condition given by the stationary solution to Eq. (12) with $C=$ const., $\epsilon_{h}=$ const. it was shown $k(\alpha)$ in Eq. (19) satisfies the relation

$$
\int_{V} \frac{\partial k(\alpha)}{\partial \alpha} d V \delta \alpha=\int_{V} 2 \alpha(1-\alpha) \nabla \cdot \mathbf{n}_{\gamma} d V \delta \alpha .
$$


As $\delta k / \delta \alpha$ in Eq. (20) does not depend explicitly on $\epsilon_{h}(\mathbf{x}, t)$ the above relation will be also used herein.

Next it is proven the functional derivative of Eq. (19) with the variable characteristic length scale $\epsilon_{h}(\mathbf{x}, t)$ leads to the stationary solution of Eq. (12) accounting for the non-equilibrium effects. The RHS of stationary Eq. (12) leads to re-initialization equation in the non-conservative form

$$
\frac{\partial \alpha}{\partial \tau}=\epsilon_{h} \nabla^{2} \alpha+\nabla \epsilon_{h} \cdot \nabla \alpha-\frac{\alpha(1-\alpha)}{\epsilon_{h}}\left[(1-2 \alpha)+\epsilon_{h} \nabla \cdot \mathbf{n}_{\gamma}\right]
$$

where we set $C=1[\mathrm{~m} / \mathrm{s}]$ for clarity. Calculation of the functional derivative of Eq. (19) with $\epsilon_{h}(\mathbf{x}, t)$ is carried out in Appendix B. The minimization condition given by Eq. (B.6) is the same as the RHS of Eq. (21), therefore

$$
\frac{\delta F}{\delta \alpha}=\frac{\partial \alpha}{\partial \tau}=0
$$

The stationary solution to Eq. (12) or the steady state solution of the corresponding re-initialization equation in pseudo-time $\tau$ would minimize the functional (19) with the variable characteristic length scale $\epsilon_{h}(\mathbf{x}, t)$. The additional term $\nabla \epsilon_{h} \cdot \nabla \alpha$ forces changes of the function $\alpha$ shape. When $\epsilon_{h}=$ const. the equilibrium solution given by Eqs. (13) and (14) is recovered. In the following section, the mapping function used during numerical solution of Eqs. (17) and (18) with variable $\epsilon_{h}(\mathbf{x}, t)$ is derived.

\subsection{Modification of the mapping procedure}

In the present section it is proposed how to use the re-initialization equation in the form of Eq. (18) taking into account variable $\epsilon_{h}(\mathbf{x}, t)$. The equilibrium condition obtained from the stationary solution to Eq. (12) reads

$$
\nabla \alpha=|\nabla \alpha| \mathbf{n}_{\gamma}=\frac{\alpha(1-\alpha)}{\epsilon_{h}(\mathbf{x}, t)} \mathbf{n}_{\gamma}
$$

Eq. (23) is formulated in the direction $\mathbf{n}_{\gamma}$ normal to the regularized interface $\gamma$, hence, it may be rewritten as

$$
\frac{\partial \alpha}{\partial \psi}\left|\frac{\partial \psi}{\partial \mathbf{x}}\right|=\frac{1}{\epsilon_{h}(\mathbf{x}, t)} \alpha(1-\alpha)
$$


where it is assumed $\partial \alpha / \partial \psi>0$ meaning $\alpha(\psi)$ is expected to be the cumulative distribution function with infinite support analogously to Eq. (15). Next, we assume $|\nabla \psi| \equiv 1$ in Eqs. (23) and (24). As a result, substitution of Eq. (23) into Eq. (12) with $D(\mathbf{x}, t)=C \epsilon_{h}(\mathbf{x}, t)$ let us derive Eq. (18). The assumption $|\nabla \psi| \equiv 1$ means the signed distance function $\psi(\mathbf{x}, t)$ spans the space where surface averaged oscillations of the sharp interface $\Gamma$ take place. On average, these oscillations occur only in the direction $\mathbf{n}_{\gamma}$ normal to the expected position $\psi=0$ of the regularized interface $\gamma$. The above interpretation explains the difference between $\psi(\alpha)$ and $\Psi(\mathbf{x}, t)$ signed distance function fields. $\Psi(\mathbf{x}, t)$ is exclusively the signed distance from points $\delta_{\Gamma}(\Psi)$ located at the sharp interface $\Gamma$ defined by the level-set $\Psi(\mathbf{x}, t)=0$.

Further, it is noticed at each point $(\mathbf{x}, t)$ of the field $\epsilon_{h}(\mathbf{x}, t)$ the signed distance function $\psi(\mathbf{x}, t)$ is given. Hence, the knowledge of the field $\psi(\mathbf{x}, t)$ gives $(\mathbf{x}, t)$ and thus $\epsilon_{h}(\mathbf{x}, t)$. Therefore, we introduce $\epsilon_{h}^{\psi}(\mathbf{x}, t)$ denoting $\epsilon_{h}(\mathbf{x}, t)$ determined using $\psi(\mathbf{x}, t)$. This let us to integrate Eq. (24) in the local coordinate system attached to the regularized interface $\gamma$. As $\gamma$ is defined by $\psi(\mathbf{x}, t)=0$, $\psi(\mathbf{x}, t)$ is the normal coordinate with the origin at $\psi(\mathbf{x}, t)=0$ of this local system. At each fixed point of given $\alpha(\psi), \psi(\alpha), \epsilon_{h}(\mathbf{x}, t)$ fields this integration reads

$$
\int_{\alpha(\psi)}^{1 / 2} \frac{d \alpha^{\prime}}{\alpha^{\prime}\left(1-\alpha^{\prime}\right)}=\int_{\psi(\alpha)}^{0} \frac{d \psi^{\prime}}{\epsilon_{h}^{\psi^{\prime}}(\mathbf{x}, t)} .
$$

The integration (25) is performed from the arbitrary point located at the signeddistance from the regularized interface $\alpha(\psi)-\psi(\alpha)$ to the expected position of the regularized interface $\psi(\alpha=1 / 2)=0$. One notes the LHS integration in Eq. (25) does not assume or result in any specific form/shape of the function $\alpha(\psi)$.

To recover the equilibrium solution when $\epsilon_{h}(\mathbf{x}, t)=$ const. it is necessary to preserve the mapping between $\alpha(\psi)-\psi(\alpha)$, see Eq. (14). For this reason, it is more convenient to reformulate the RHS integral in Eq. (25) using variable substitution as follows

$$
\int_{\psi(\alpha)}^{0} \frac{d \psi^{\prime}}{\epsilon_{h}^{\psi^{\prime}}(\mathbf{x}, t)}=\psi(\alpha) \int_{1}^{0} \frac{d t^{\prime}}{\epsilon_{h}^{t^{\prime} \psi}(\mathbf{x}, t)}=\psi(\alpha) \mathcal{I}(\psi)
$$


where $t^{\prime} \in[0,1]$ is the parameter such that $\psi^{\prime}=t^{\prime} \psi$ and $d \psi^{\prime}=d t^{\prime} \psi$, furthermore $\mathcal{I}(\psi)$ is used to denote the integral on the RHS of Eq. (26). After integration of Eq. (24) with Eq. (26) one obtains

$$
\psi(\alpha)=\frac{1}{\mathcal{I}(\psi)} \ln \left[\frac{\alpha(\psi)}{1-\alpha(\psi)}\right] .
$$

At the given, arbitrary point $(\mathbf{x}, t)$, the signed distance $\psi(\mathbf{x}, t)$ has the known value. For this reason, at the point $(\mathbf{x}, t)$ the integral $\mathcal{I}(\psi)=$ const. and thus the inverse relation is also true

$$
\alpha(\psi)=\frac{1}{1+\exp (-\psi(\alpha) \mathcal{I}(\psi))} .
$$

The only difference between Eqs. (13) and (14) and Eqs. (28) and (27) is the latter take into account variation of $\epsilon_{h}(\mathbf{x}, t)$ in the sense of the local equilibrium condition given by Eq. (24). When the field $\epsilon_{h}(\mathbf{x}, t)=$ const., Eq. (25) and Eq. (26) reduce to the equilibrium solution, which is guaranteed by the definition of $\mathcal{I}(\psi)$. Thus, the mapping given by Eq. $(27)$ or the form of $\alpha(\psi)$ given by Eq. (28) can be employed during numerical solution of the system given by Eqs. (17) and (18) to model how the $\epsilon_{h}(\mathbf{x}, t)$ field is affecting changes of the cumulative distribution function $0<\alpha(\psi)<1$ profile.

\section{Numerical solution}

This section introduces a numerical method for the exact and approximate solutions of the intermittency region evolution equation with the structured grid solver. First, a one-dimensional study is carried out showing how the reinitialization equation (18) with the modified mapping procedure defined by Eq. (27) can be used to reconstruct the intermittency region in the non-equilibrium state. Afterwards, the coupled solution is compared with the semi-analytical approach using Eq. (18) where $\epsilon_{h}=$ const. and Eq. (28) is accounting for variable $\epsilon_{h}(\mathbf{x}, t)$. Finally, the semi-analytical solution is used in two-dimensional studies without and with advection to reconstruct more complex behavior of the intermittency region. Details of discretization and numerical solution of 
Eqs. (17) and (18) using the mapping given by Eq. (14) where $\epsilon_{h}=$ const. are described in (Wacławczyk, 2015, 2017). In Appendix D, minor modifications to these schemes required to take into account variable $\epsilon_{h}(\mathbf{x}, t)$ are described.

\subsection{Approximation of the local equilibrium condition}

The main problem during numerical solution of the set of algebraic, partial differential equations $(17,18,27)$ or $(17,18,14)$ where $\epsilon_{h}=$ const. and Eq. $(28)$ is accounting for $\epsilon_{h}(\mathbf{x}, t)$, is approximation of the integral in the local equilibrium condition given by Eq. (26). Namely, one needs to find the quadrature $I(\psi)$ for integral

$$
\mathcal{I}(\psi)=\int_{1}^{0} \frac{d t^{\prime}}{\epsilon_{h}^{t^{\prime} \psi}(\mathbf{x}, t)} \approx I(\psi)
$$

keeping in mind the parameter $t^{\prime}$ is changing along the signed distance function $\psi(\alpha)$, from the local position on the computational grid at $t^{\prime}=1$ to the interface at $t^{\prime}=0$. As function $\psi(\alpha)$ is also the solution to Eq. (18), one can use this to formulate an effective numerical integration procedure.

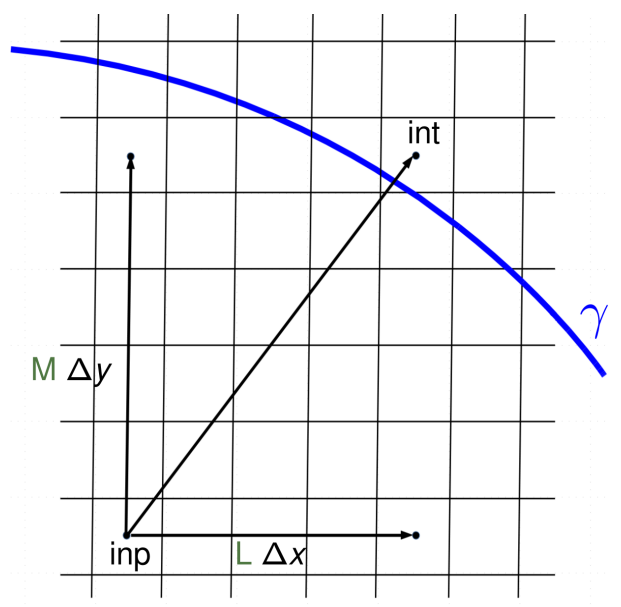

Figure 3: Determination of the interface $\gamma$ position on structured grid using the signed distance function $\psi(\alpha)$ (not shown). Schematic presentation of the points at $t^{\prime}=0$ (int) and $t^{\prime}=1$ (inp) required to compute the trapezoidal quadrature, see Eq. (30).

Let us note if the two point, first-order accurate quadrature is used, the discussed problem is reduced to finding index (int) of the control volume with 
the interface $\gamma$ during loop over all grid points with the index (inp). The sketch of this procedure in the two-dimensional case for the one pair of control volumes (inp) and (int) is depicted in Fig. 3. The simplest quadrature taking into account the two point information required to approximate Eq. (29) is given by the first-order accurate trapezoidal rule

$$
I_{T}(\psi)=\frac{1}{2}\left[\frac{1}{\epsilon_{h}(\text { int })}+\frac{1}{\epsilon_{h}(\text { inp })}\right] .
$$

To obtain a higher order of accuracy, the third-order accurate Simpson rule can be used to approximate Eq. (29) as well, it reads

$$
I_{S}(\psi)=\frac{1}{6}\left[\frac{1}{\epsilon_{h}(\text { int })}+\frac{4}{\epsilon_{h}(\text { inm })}+\frac{1}{\epsilon_{h}(\text { inp })}\right],
$$

where (inm) denotes control volume in the center between (inp), (int). In what follows it is compared how approximations of the integral (29) given by Eqs. (30) and (31) affect the obtained solutions. Introduction of an even higher order of accuracy in the approximation of Eq. (29) requires considering additional control volumes in-between the local position on the mesh (inp) and the expected position of the interface $\gamma$ (int). When the number of control volumes between (inp) and (int) is smaller than the quadrature stencil, the higherorder quadrature has to be replaced by the appropriate lower-order quadrature or interpolation of $\epsilon_{h}(\mathbf{x}, t)$.

As the present results are obtained using the structured grid solver, computation of Eq. (30) or Eq. (31) is straightforward. Knowing the local position at the grid (inp) and the value of the signed distance function in this cell $\psi($ inp $)$ one needs to project it on $(x, y)$ directions to obtain: $\psi_{x}=-\psi($ inp $) n_{\gamma, x}$, $\psi_{y}=-\psi(\operatorname{inp}) n_{\gamma, y}$. Next, compute constants (L, M) (see Fig. 3) where $L \approx$ $\operatorname{NINT}\left(\psi_{x} / \Delta x\right)$ and $M \approx N I N T\left(\psi_{y} / \Delta y\right)$, and finally determine the index of the cell containing interface (int), on structured grid $i n t=i n p+L N J+M$, where $N J$ is the number of grid cells in $j$ direction, NINT is the intrinsic function returning nearest integer. Point (inm) in Eq. (31) is obtained in a similar way taking $\psi_{x}^{m}=-0.5 \psi($ inp $) n_{\gamma, x}, \psi_{y}^{m}=-0.5 \psi($ inp $) n_{\gamma, y}$ and then computing $\left(L^{m}, M^{m}\right)$. If the stencil where Eq. (31) is computed is smaller than three control volumes, 
the $\epsilon_{h}$ (inm) value is obtained as $\epsilon_{h}$ (inm) $=\left(\epsilon_{h}\right.$ (inp) $+\epsilon_{h}$ (int))/2. Due to introduction of $\epsilon_{h}^{\psi^{\prime}}(\mathbf{x}, t)$ in Eq. (25), in one and three dimensional cases this procedure can be easily adopted by taking into account one less or one more spatial direction to compute (int) and/or (inm).

\subsection{Evolution of one-dimensional cumulative distribution function}

To compare the exact and approximate semi-analytical solutions and estimate numerical accuracy of the procedure introduced in Section 4.1 the evolution of the one-dimensional $\alpha(\psi)$ profile disturbed by variable $\epsilon_{h}(\mathbf{x}, t)$ is studied on three, gradually refined grids $m_{i}=2^{5+i} \times 2^{5+i}, i=1,2,3$. The characteristic length scale $\epsilon_{h}(\mathbf{x}, t)$ is predefined as the step $\epsilon_{h, S}(\mathbf{x}, t)$ or bell $\epsilon_{h, B}(\mathbf{x}, t)$ shaped disturbance, see Eqs. (C.1) and (C.2) respectively; only Eq. (18) is solved, as $\mathbf{w}=\mathbf{u}=0$ advection is neglected. The number of physical time steps is set to $N_{t}=72, \Delta t=10^{-3}[s]$. The number of re-initialization time steps, if not stated otherwise, is set to $N_{\tau}=256$ with the size $\Delta \tau=\epsilon_{h, b} / 2$ to guarantee the steady state solution of Eq. (18) after each time iteration it. The minimum (base) thickness of the interface is set to $\epsilon_{h, b}=\Delta x_{i}, i=1,2,3$. The discretization of Eq. (18) is the same as in (Wacławczyk, 2017), the only modification accounting for $\epsilon_{h}(\mathbf{x}, t)$ is introduced to the constrained interpolation used to approximate $\tilde{\delta}(\alpha)=\alpha(1-\alpha)$ in Eq. (18), see Eq. (D.5).

Figs. $4-5$ present, respectively, the evolution of $\epsilon_{h, S}(\mathbf{x}, t), \epsilon_{h, B}(\mathbf{x}, t)$ profiles and corresponding variations of $\alpha(\psi)$ at equal time intervals on the mesh $m_{2}$. The results presented therein are obtained after each time iteration it at the end of re-initialization process, see Fig. 7. The black-dashed lines in Figs. 4 - 5 represent the analytical $\alpha(\psi)$ profiles obtained using Eq. (13) with $\epsilon_{h}=$ $\epsilon_{h, b}$ (dashed line), and $\epsilon_{h}=2 \epsilon_{h, b}$ (dashed-dotted line). Therein, $\alpha_{d c}$ denotes solutions obtained using the direct coupling of Eqs. (18) and (27), $\alpha_{d}$ denotes an approximate, semi-analytical solution obtained using Eq. (18) where $\epsilon_{h}=\epsilon_{h, b}$ and Eq. (28) accounting for $\epsilon_{h}(\mathbf{x}, t)$. In both cases the third-order accurate Simpson rule (31) is used to approximate $\mathcal{I}(\psi)$ integral in Eq. (26).

The convergence space of the re-initialization equation (18) for the coupled 

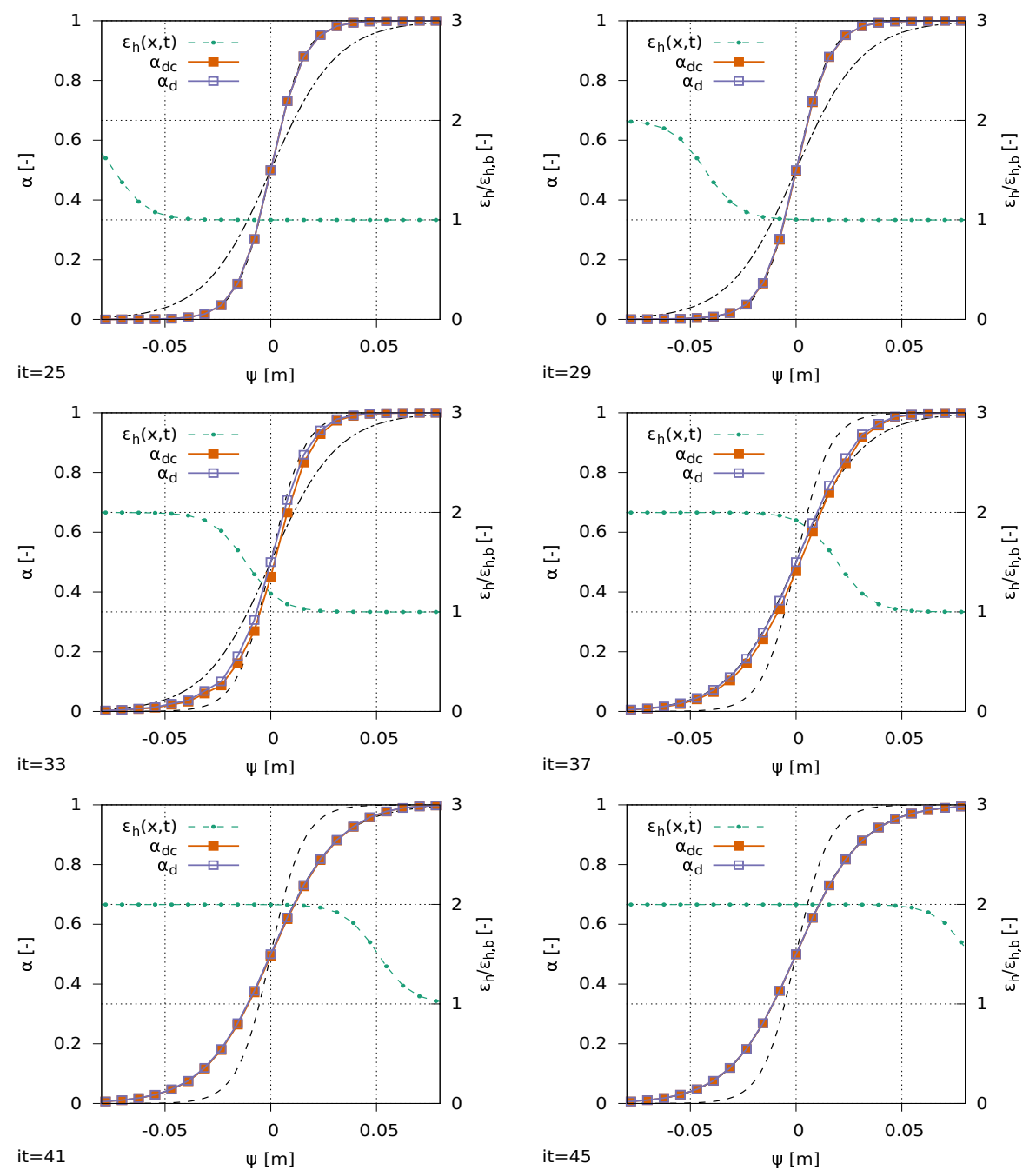

Figure 4: Comparison of $\alpha(\psi)$ affected by the step shaped variation $\epsilon_{h, S}(\mathbf{x}, t)$ defined by Eq. (C.1) shown after (left to right) equal time intervals; $\alpha_{d c}$ denotes the coupled and $\alpha_{d}$ semi-analytical solution, respectively. Black-dashed lines mark the analytical profiles of $\alpha(\psi)$ where $\epsilon_{h}=\epsilon_{h, b}$ or $\epsilon_{h}=2 \epsilon_{h, b}$, see Eq. (13).

$\alpha_{d c}$ cases is presented in Figs. $6-8$ on the meshes $m_{i}=2^{5+i} \times 2^{5+i}, i=1,2,3$ respectively. Therein, the $L_{1, \tau}$ norm defined by Eq. (E.1) characterizing the numerical solution of Eq. (18) in times $t, \tau$ is presented. The top row shows convergence of Eq. (18) obtained using the first-order accurate quadrature (30), 
the bottom row using the third-order accurate quadrature (31). Although in
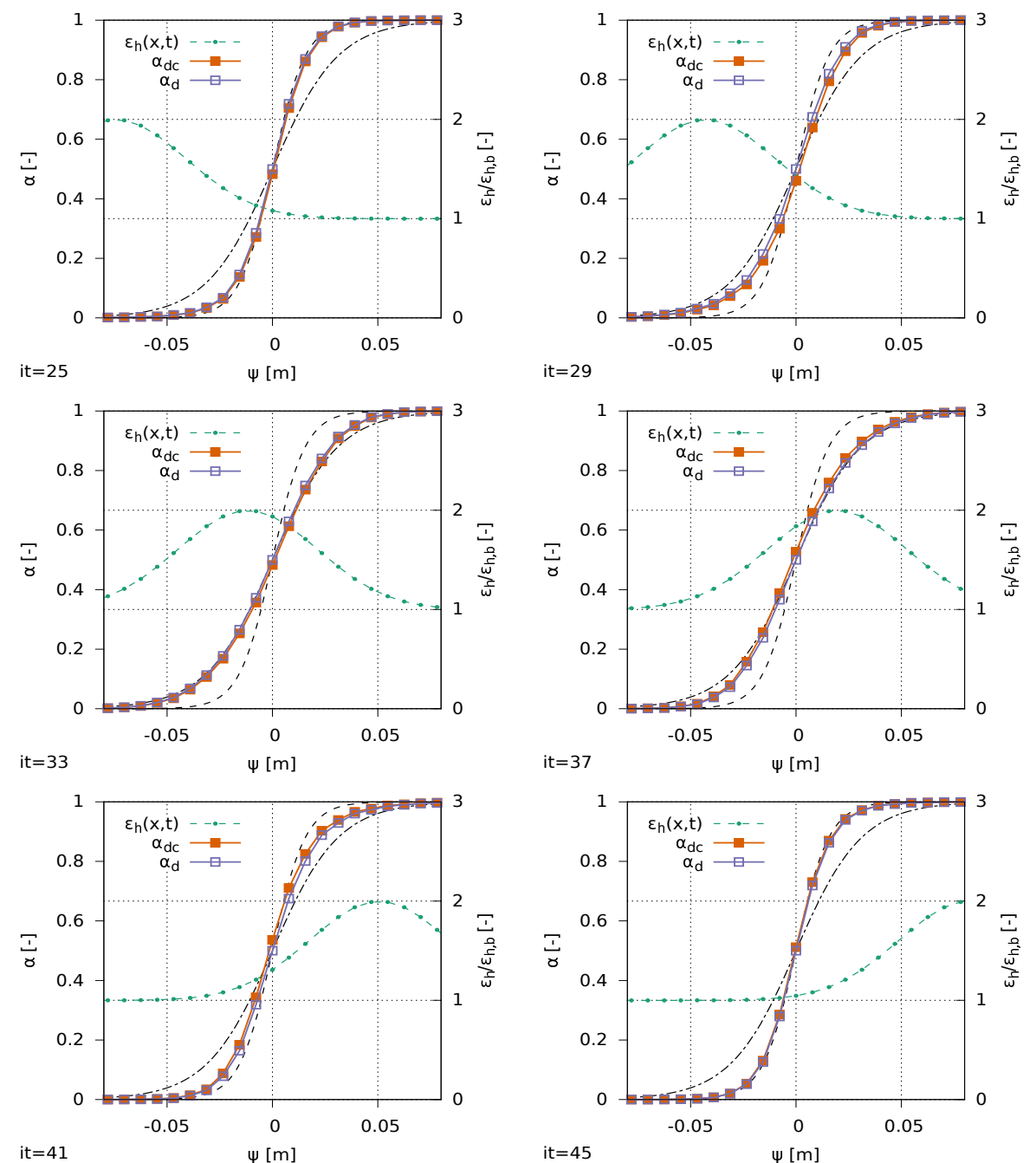

Figure 5: Comparison of $\alpha(\psi)$ affected by the bell shaped variation $\epsilon_{h, B}(\mathbf{x}, t)$ defined by Eq. (C.2), shown after (left to right) equal time intervals; $\alpha_{d c}$ denotes the coupled and $\alpha_{d}$ semi-analytical solution, respectively. Black-dashed lines mark the analytical profiles of $\alpha(\psi)$ where $\epsilon_{h}=\epsilon_{h, b}$ or $\epsilon_{h}=2 \epsilon_{h, b}$, see Eq. (13).

all cases the convergence of the numerical solution is obtained, one observes the variation of $\alpha(\psi)$ caused by $\epsilon_{h}(\mathbf{x}, t)$ strongly affects the numerical solution of Eq. (18). In order to avoid this dependence, the approach where $\epsilon_{h}=\epsilon_{h, b}=$ const. 
in Eq. (18) and $\epsilon_{h}(\mathbf{x}, t)$ variation is modeled using Eq. (28) is introduced. In this case, the $L_{1, \tau}$ norm remains almost constant $\sim 10^{-16}$ for all steps $t, \tau$ (Wacławczyk, 2015); $\psi$ field obtained during the solution of Eq. (18) where $\epsilon_{h}=\epsilon_{h, b}$ is treated as the carrier function for $\alpha(\psi)$ given by Eq. (28).

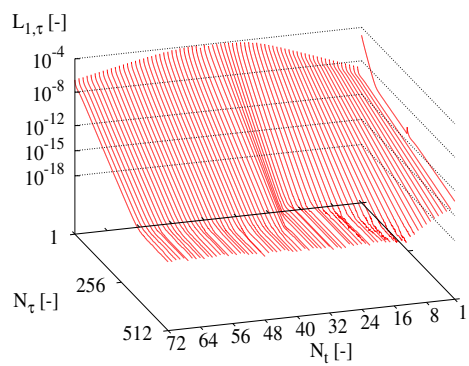

(a)



(c)

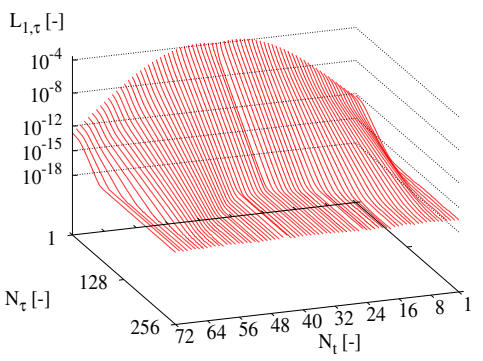

(b)

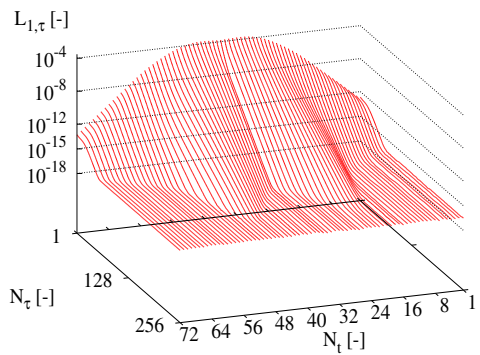

(d)

Figure 6: Convergence space of $L_{1, \tau}$ norm given by Eq. (E.1), during solution of Eq. (18) and Eq. (27) on the mesh $m_{1}$. The one-dimensional $\alpha(\psi)$ profile is altered by $\epsilon_{h, S}(\mathbf{x}, t)$ (left column) or $\epsilon_{h, B}(\mathbf{x}, t)$ (right column) see Figs. $4-5$, respectively. The results are obtained with $N_{t}=72$ time and $N_{\tau}=512(\mathrm{a}, \mathrm{c})$ or $N_{\tau}=256(\mathrm{~b}, \mathrm{~d})$ re-initialization steps; the trapezoidal (top row) or Simpson (bottom row) rules are employed to approximate integral in Eq. (26).

The differences between $\alpha_{d c}$ and $\alpha_{d}$ profiles observed in Figs. $4-5$ are the consequence of differences in $\psi$ fields obtained during the coupled and semianalytical solutions. During the direct coupling, $\psi(\alpha)$ is part of the numerical 


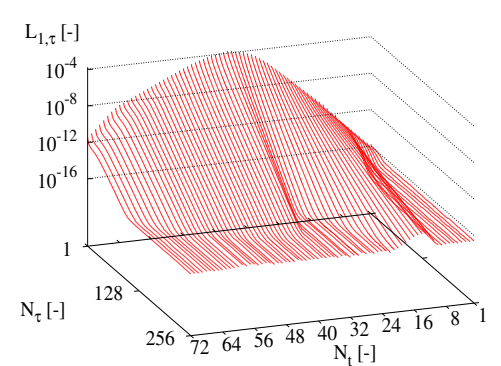

(a)

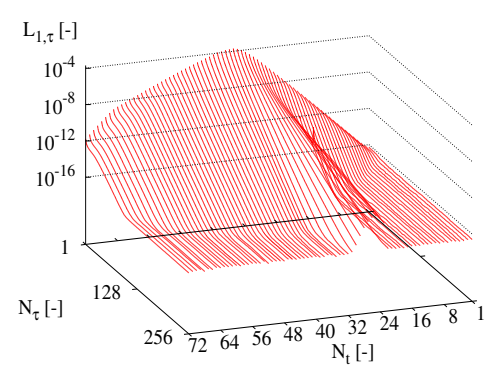

(c)

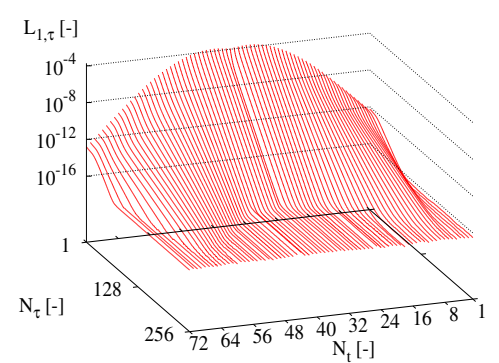

(b)

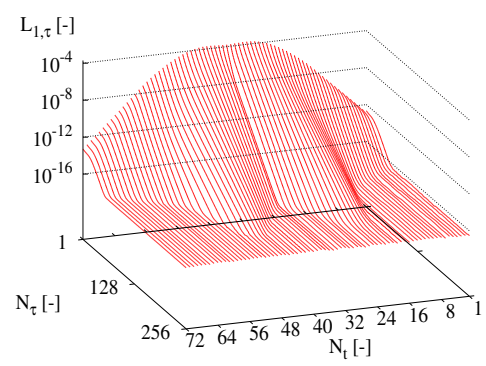

(d)

Figure 7: Convergence space of $L_{1, \tau}$ norm given by Eq. (E.1), during solution of Eq. (18) and Eq. (27) on the mesh $m_{2}$. The one-dimensional $\alpha(\psi)$ profile is altered by variable $\epsilon_{h, S}(\mathbf{x}, t)$ (left column) or $\epsilon_{h, B}(\mathbf{x}, t)$ (right column). The results are obtained with $N_{t}=72$ time and $N_{\tau}=256$ re-initialization steps; the trapezoidal (top row) or Simpson (bottom row) rules are employed to approximate integral in Eq. (26).

solution and hence the expected position of the regularized interface $\psi=0$ can change its location. In the semi-analytical case, $\epsilon_{h}=\epsilon_{h, b}=$ const., for this reason the position $\psi=0$ is not affected by variations of the characteristic length scale field $\epsilon_{h}(\mathbf{x}, t)$. The latter approach simplifies numerical solution of Eq. (18), but still the semi-analytical solution $\alpha_{d}$ closely mimics the exact one $\alpha_{d c}$, compare results in Figs. $4-5$.

Fig. 9 illustrates how the order of accuracy of the quadrature used in Eq. (26) affects the numerical results obtained on the grid $m_{1}$. Therein it can be observed 


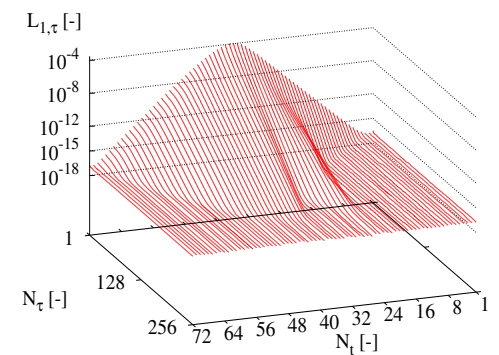

(a)

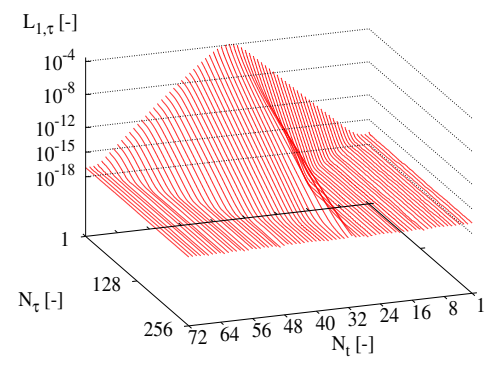

(c)

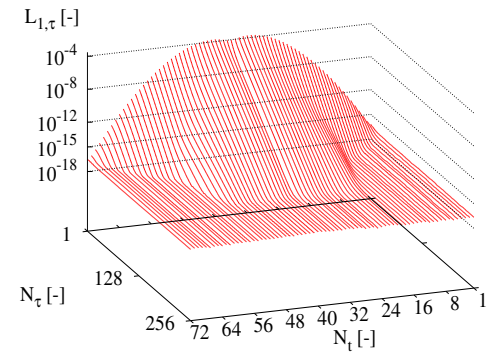

(b)

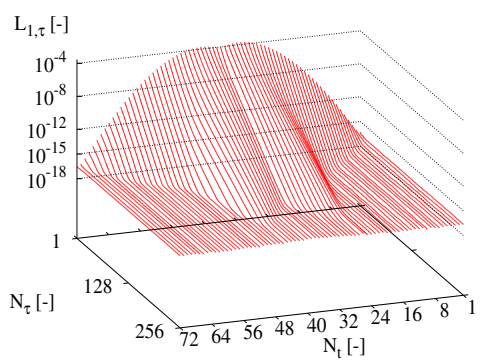

(d)

Figure 8: Convergence space of $L_{1, \tau}$ norm given by Eq. (E.1), during solution of Eq. (18) and Eq. (27) on the mesh $m_{3}$. The one-dimensional $\alpha(\psi)$ profile is altered by variable $\epsilon_{h, S}(\mathbf{x}, t)$ (left column) or $\epsilon_{h, B}(\mathbf{x}, t)$ (right column). The results are obtained with $N_{t}=72$ time and $N_{\tau}=256$ re-initialization steps; the trapezoidal (top row) or Simpson (bottom row) rules are employed to approximate integral in Eq. (26).

the quadrature selection has an notable but small impact on the obtained results. Differences between fully coupled solutions reconstructed using the first-order trapezoidal $\alpha_{T, d c}$ or third-order accurate Simpson $\alpha_{S, d c}$ rules (solid symbols) are almost the same as differences between analogical semi-analytical solutions: $\alpha_{T, d}$ and $\alpha_{S, d}$ (hollow symbols), compare results in Fig. 9. The deviations between the results obtained using first- or third-order accurate quadrature are most pronounced in the regions where the slope change of the $\epsilon_{h}(\mathbf{x}, t)$ profile is significant. This is expected in view of the definition of the order of accuracy 

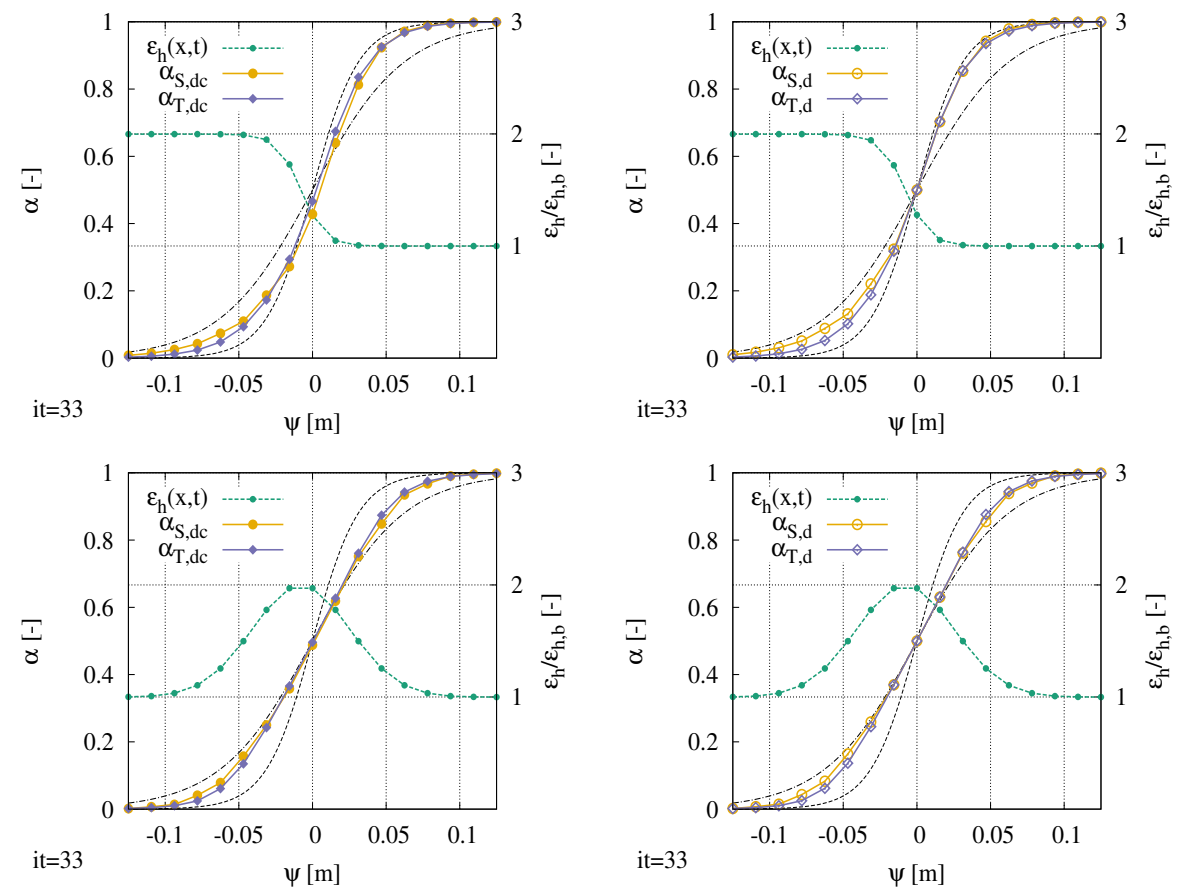

Figure 9: Comparison of $\alpha(\psi)$ profiles affected by the step (top row) or bell (bottom row) shaped variation of the $\epsilon_{h}(\mathbf{x}, t)$ field. Results are predicted using the trapezoidal $\left(\alpha_{T}\right)$ or Simpson rules $\left(\alpha_{S}\right)$ in the case of direct $\left(\alpha_{d c}\right)$ or semi-analytical $\left(\alpha_{d}\right)$ solutions at two selected time moments $i t \cdot \Delta t$ using the grid $m_{1}$. Black dashed lines mark the analytical solution given by Eq. (13) where $\epsilon_{h}=\epsilon_{h, b}$ or $\epsilon_{h}=2 \epsilon_{h, b}$, respectively.

of the both integration rules.

The impact of $\epsilon_{h}(\mathbf{x}, t)$ on $\alpha(\psi)$ studied in Figs. $4-5$ can be summarized as follows. As it is anticipated, variations of $\epsilon_{h}(\mathbf{x}, t)$ affect the shape of the cumulative distribution function $\alpha(\psi)$. In the case of asymmetric, step shaped $\epsilon_{h, S}$ this ultimately leads to the increase of the width of the intermittency region, see Fig. 4. One observes the $\alpha(\psi)$ profile approaches the equilibrium, analytical solution given by Eq. (13) with $\epsilon_{h}=2 \epsilon_{h, b}$, see Fig. $4 i t=45$. We note that in the present numerical procedure the width of the intermittency region is constrained by $\epsilon_{h, b}$, hence, the variation of $\epsilon_{h}$ can not result in the intermittency region being thinner than $\epsilon_{h, b}$. In Fig. 4, the approximate solution $\alpha_{d}$ is reacting slightly 
faster on $\epsilon_{h, S}$ variation than $\alpha_{d c}$. However, $\alpha_{d}$ follows the direct solution $\alpha_{d c}$ very closely. The solutions $\alpha_{d c}, \alpha_{d}$ are bounded by the analytical $\alpha(\psi)$ profiles (black-dashed lines) with the extreme values of $\epsilon_{h}=\epsilon_{h, b}$ and $\epsilon_{h}=2 \epsilon_{h, b}$.

The same conclusions can be drawn from the results presented in Fig. 5. During the non-symmetric changes of $\alpha(\psi)$ both, the exact and approximate solutions display similar behavior, initially resulting in the increase and then decrease of the intermittency region width. After the peak of $\epsilon_{h, B}(\mathbf{x}, t)$ passes the expected position of the interface at $\psi=0$ the analytical $\alpha(\psi)$ profile with $\epsilon_{h}=\epsilon_{h, b}$ is recovered, see Fig. 5, it $=45$. It is noted, the return of $\alpha(\psi)$ to the equilibrium state is guaranteed by design of the mapping function Eq. (27) and quadrature in Eq. (29).

The history of convergence of the numerical error on the three gradually refined grids $m_{i}, i=1,2,3$ is presented in Fig. 10. It shows $L_{1, S}(\alpha)$ and $L_{1, B}(\alpha)$ norms defined by Eq. (E.2) plotted after each time iteration $i t=1, \ldots, 72$. We note that the differences in the numerical error of the semi-analytical solutions (hollow symbols in Fig. 10(a,c) and Fig. 10(b,d)) are only caused by the errors of the quadrature used to approximate Eq. (26).

Convergence of the solution is obtained on each grid $m_{i}, i=1,2,3$ what is confirmed by the results depicted in Figs. $6-8$. When the Simpson rule is used to approximate the integral in Eq. (26) the level of the numerical errors is lower in comparison with the trapezoidal rule, compare the results in the bottom and top row in Fig. 10. The errors of the coupled $L_{1, S}^{d c}, L_{1, B}^{d c}$ (solid symbols) and semi-analytical $L_{1, S}^{d}, L_{1, B}^{d}$ (hollow symbols) solutions display similar behavior; the error of the coupled solution is always higher than the error of the respective semi-analytical solution, see Fig. 10(a,b,c,d). One notes that in the case $\epsilon_{h}=\epsilon_{h, S}$ when the trapezoidal quadrature is used on the grid $m_{1}$ (see Fig. 10(a)) the coupled and semi-analytical solutions are different opposite to the case when the Simpson rule is employed, compare $m_{1, d c}, m_{1, d}$ in Fig. 10(a,c). In the case $\epsilon_{h}=\epsilon_{h, B}$ the differences between $L_{1, B}^{d c}$ (solid symbols) and $L_{1, B}^{d}$ (hollow symbols) can be spotted only on the grid $m_{3}$ at the beginning it $<4$ and at the end of simulation it $>68$, see $m_{3, d c}, m_{3, d}$ in Fig. $10(\mathrm{~b}, \mathrm{~d})$. 


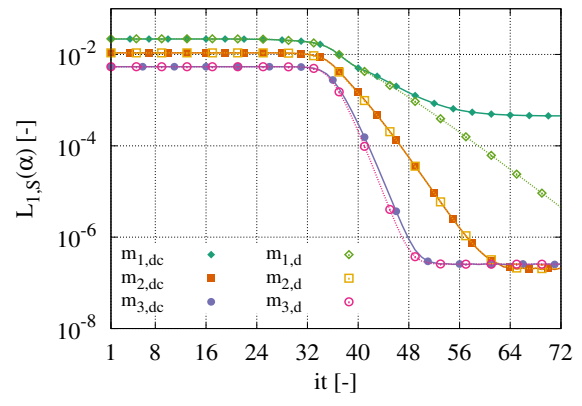

(a)

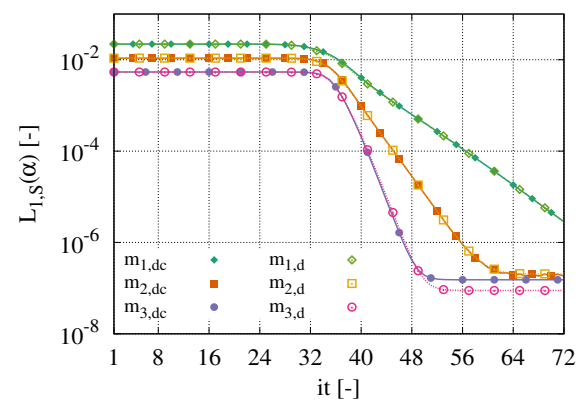

(c)

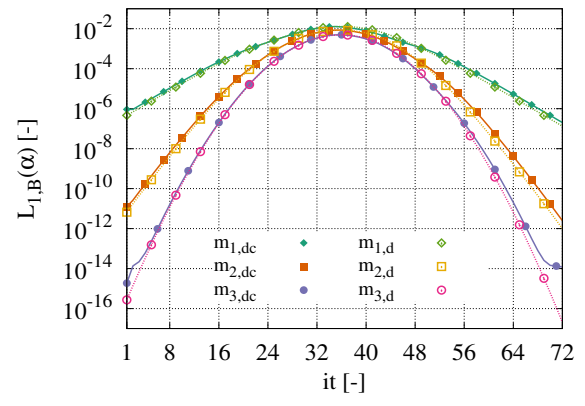

(b)

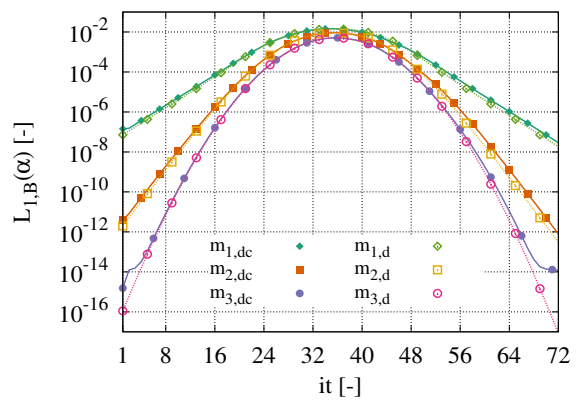

(d)

Figure 10: Temporal evolution of the $L_{1, S}(\alpha)$ and $L_{1, B}(\alpha)$ norms defined by Eq. (E.2) in physical time $i t \cdot \Delta t$. The error of the coupled solution (dc) (Eqs. (18) and (27)) is marked with the solid symbols, the error of the semi-analytical solution (d) (Eq. (28)) is marked with the hollow symbols; $\epsilon_{h}=\epsilon_{h, S}$ (left column) or $\epsilon_{h}=\epsilon_{h, B}$ (right column). The integral in Eq. (29) is approximated using the trapezoidal (top row) or Simpson (bottom row) rules, respectively.

To stabilize the numerical solution of Eqs. (18) and (27) on the grid $m_{1}$ when $\epsilon_{h}=\epsilon_{h, S}$ it was necessary to reduce the time step size $\Delta \tau$ and increase the number of re-initialization steps $N_{\tau}$, see results in Fig. 6(a,c). In this case $N_{\tau}=512$ and $\Delta \tau=0.25 \Delta x$, for both integration rules used.

Some instabilities in convergence of the numerical solutions on the grid $m_{1}$, $m_{2}$ can be seen in Fig. 6(a,c) and Fig. 7(c). However, they are present only in the case $\epsilon_{h}=\epsilon_{h, S}$ and vanish with increasing spatial resolution, compare results in Figs. $7(\mathrm{a}, \mathrm{c})-8(\mathrm{a}, \mathrm{c})$. It is emphasized that the algorithm employed 


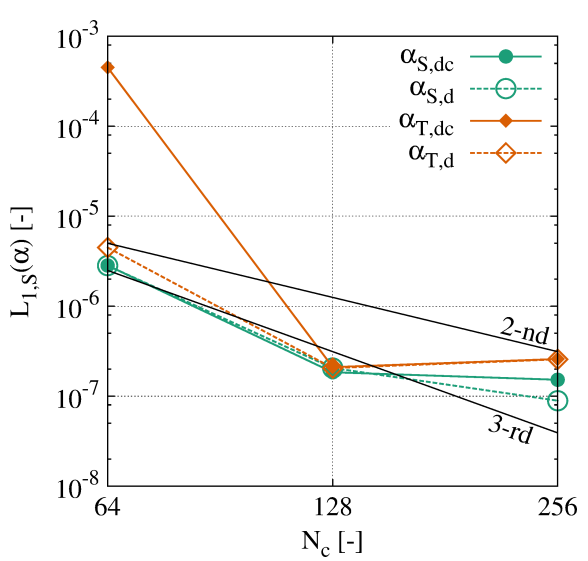

(a)

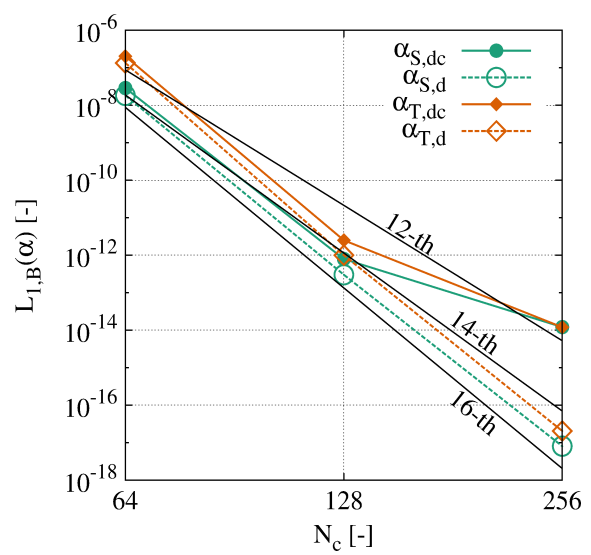

(b)

Figure 11: Spatial convergence rates of the numerical errors $L_{1, S}, L_{1, B}$ defined by Eq. (E.2) at $i t=72$, see Fig. 10; (a) $\epsilon_{h}=\epsilon_{h, S}$, (b) $\epsilon_{h}=\epsilon_{h, B}$. Figure shows the convergence rate of the coupled $\alpha_{d c}$ (solid symbols) and semi-analytical $\alpha_{d}$ (hollow symbols) solution. The intergral in Eq. (26) is discretized using the trapezoidal $(T)$ or Simpson $(S)$ rules.

in the present work to reconstruct solution of Eqs. (18) and (27) does not use flux limiters or non-oscillatory schemes in order to stabilize convergence of the numerical solution.

On the grid $m_{1}$ when $\epsilon_{h}=\epsilon_{h, S}$, the first- or third-order accuracy is not sufficient to obtain the truncation error level after all physical time steps it after predefined $N_{\tau}=512$ re-initialization steps, see Fig. 6(a,c). For this reason, the norm $L_{1, S}(\alpha)$ (see $m_{1}$ results in Fig. $10(\mathrm{a}, \mathrm{c})$ ) does not converge to the stationary state, too. In particular, this is visible in the semi-analytical solution, see hollow symbols in Fig. 10(a,c). This might be caused by not fully converged computations at the time iterations $1<i t<16$ (see Fig. $6($ a)) and $8<i t<32$ (see Fig. 6(c)) . We note similar behavior of the numerical error is observed when grid $m_{2}$ is used, see Fig. 7 (c) $12<i t<24$. However therein, the error level is approximately five orders of the magnitude lower than in Fig. 6(a,c) and the convergence to the stationary state is achieved with both integration rules, see results $m_{2, d c}, m_{2, d}$ in Fig. 10(a,c). 
Interestingly, in the case of $\epsilon_{h}=\epsilon_{h, B}$ the problem described above does not show up, see the right column in Fig. 10. Both quadratures in the coupled (solid symbols) and semi-analytical (hollow symbols) solutions predict similar evolution of the numerical error $L_{1, B}(\alpha)$, see Fig. 10(b,d). A cursory explanation for this result is that in the present study spatial dimensions of $\epsilon_{h}(\mathbf{x}, t)$ are constant on all used grids $m_{i}$ (count the number of grid points in $\epsilon_{h}(\mathbf{x}, t)$ profile in Fig. 9, $i t=33$ and in Fig. 4, $i t=33$ ). Thus on the grid $m_{1}$ the step profile $\epsilon_{h, S}(\mathbf{x}, t)$ is represented by four grid points and this impairs solution of Eq. (27). This reckoning is confirmed by the convergence rates of the spatial discretization error presented in Fig. 11 . Therein, the $L_{1, S}(\alpha)$ and $L_{1, B}(\alpha)$ norms depicted in Fig. 10 are plotted at the time moment $t=72 \cdot \Delta t$. One notes that the spatial convergence rate is strongly affected by the choice of the shape of $\epsilon_{h}(\mathbf{x}, t)$ profile and the order of accuracy of the quadrature has little impact on the $L_{1, S}(\alpha), L_{1, B}(\alpha)$ norms convergence rates, compare solid and hollow symbols in Fig. 11(a,b).

Fig. 11(a) shows that in the case $\epsilon_{h}=\epsilon_{h, S}$ the convergence rate of the coupled (dc) and semi-analytical (d) solution is second-order accurate for both quadratures used. As the semi-analytical solution introduces lower discretization error the level of $L_{1, S}$ norm for $\alpha_{S, d}, \alpha_{T, d}$ is lower than in the coupled case, see Fig. 11(a). When $\alpha(\psi)$ is affected by $\epsilon_{h, B}$ the high-order of the convergence rate of all numerical solutions is obtained. The first-, and third-order accurate quadrature reconstruct the numerical solution with the same convergence rate. The error level of the coupled solution is higher than the error level of the semi-analytical solution, see Fig. 11(b).

At first, high-orders of the convergence rate presented in Fig. 11 appear to be unexpected. However, in the present study $\epsilon_{h, b}=\Delta x_{i}, i=1,2,3$ this guarantees the resolution in the one-dimensional case is four times higher than in the previous papers of the present author (Wacławczyk, 2015, 2017) where the convergence of Eq. (18) was investigated. Moreover, we note herein solution of Eq. (18) in time $\tau$ is carried out until the level of the truncation error is achieved in the double precision computations, see Figs. $6-8$. In aforementioned works 
the tests with advection were carried out using $N_{\tau}=4$ (Wacławczyk (2017) see Fig. 7, 9) or $1<N_{\tau}<16$ (Wacławczyk (2015) see Fig. 31) re-initialization steps. Yet other argument supporting results in Fig. 11(b) is the present method is based on the semi-analytical solution of the set of partial differential algebraic equations $(17,18,27)$, see Section 4.1 and Appendix D. This is the main difference between the present semi-numerical approach and purely numerical techniques (VOF, SLS methods) known in the literature.

The results presented in Figs. $4-5$ and convergence study presented in Figs. $10-11$ confirm the semi-analytical solution $\alpha_{d}$ is providing close estimation of the coupled problem $\alpha_{d c}$. In the next section this solution is used to model variation of the intermittency region around circular drop without and with advection. As the third-order accurate Simpson quadrature (31) is more sensitive to variations of $\epsilon_{h}(\mathbf{x}, t)$ it is used in all the following numerical tests.

\subsection{Two-dimensional semi-analytical solution}

To assess how the numerical method introduced in Section 4.1 works in the two-dimensional case, the resting, circular drop centered at the point $(0.5,0.5)$ with radius $R_{B}=0.15[m]$ surrounded by the intermittency region and disturbed by the $\epsilon_{h, B} \leq \epsilon_{h}(\mathbf{x}, t) \leq 5 \epsilon_{h, B}$ field is studied, see Fig. 12. In this test, $\epsilon_{h}(\mathbf{x}, t)$ is evolving according to Eq. (C.3). The problem is solved in a two-dimensional unit square box $[0,1] \times[0,1]$ discretized with $2^{8} \times 2^{8}$ control volumes; the base width of the intermittency region is set to $\epsilon_{h, b}=\sqrt{2} \Delta x / 4[m]$, the physical time step size is $\Delta t=10^{-3}[s]$, fictitious time step size $\Delta \tau=\epsilon_{h, b} / 2[s]$, four re-initialization steps $N_{\tau}=4$ per $\Delta t$ are used. Only Eq. (18) with $\epsilon_{h, b}=$ const. and Eq. (28) taking into account $\epsilon_{h}(\mathbf{x}, t)$ are solved as $\mathbf{w}=\mathbf{u}=0$.

The evolution of the intermittency region due to variable $\epsilon_{h}(\mathbf{x}, t)$ is illustrated in Figs. 12 - 13. Fig. 12 displays variation of the $\epsilon_{h}(\mathbf{x}, t)$ field and its impact on $\alpha(\psi)$ illustrated using contours $\alpha\left(\psi=-4 \epsilon_{h}\right), \alpha(\psi=0), \alpha\left(\psi=4 \epsilon_{h}\right)$. The variation of $\epsilon_{h}(\mathbf{x}, t)$ in the subsequent time moments $i t \cdot \Delta t$ leads first to an increase and later a decrease of the intermittency region width (similarly to the one-dimensional predictions in Fig. 5). Details of this process can be observed 



Figure 12: Evolution of the intermittency region affected by the variable, defined by Eq. (C.3) field of $\epsilon_{h, b} \leq \epsilon_{h}(\mathbf{x}, t) \leq 5 \epsilon_{h, b}$ (colors); all figures show contours $\alpha(\psi=$ $\left.-4 \epsilon_{h, b}\right), \alpha(\psi=0), \alpha\left(\psi=4 \epsilon_{h, b}\right)$.

in Fig. 13. Therein, $\epsilon_{h}(\mathbf{x}, t)$ and $\alpha(\psi)$ profiles are drawn along the parts $R 1$, $R 2$ of the diagonal across the computational domain (see Fig. 12, it $=12$ ) at seven different time moments $i t \cdot \Delta t$. Fig. 13a,b is illustrating variations in the region $R 1$ and Fig. 13c,d in the region $R 2$.

In Fig. 13(a), the impact of $\epsilon_{h}(\mathbf{x}, t)$ increasing in time on $\alpha(\psi)$ is presented. It can be observed the profile of $\alpha(\psi)$ converges to analytical solution given by Eq. (13) with $\epsilon_{h}=2.5 \epsilon_{h, b}$ (not shown in Fig. 13 for clarity of presentation). After the bell shaped disturbance (moving to the left) passes $\psi=0$ (near $i t=36$ ) the process is reversed and at $i t=60$ the equilibrium solution with $\epsilon_{h}=\epsilon_{h, b}$ is reconstructed, see Fig. 13b. Variation of $\epsilon_{h}(\mathbf{x}, t)$ in the region $R 2$ is more complex, see Fig. 13c,d. Therein, the step (moving to the left) and bell (moving to the right) shaped disturbances interfere, leading to increased $\alpha(\psi)$ profile width close to the one obtained when $\epsilon_{h}=5 \epsilon_{h, b}$ in Eq. (13), see Fig. 13c, $i t=36$. In the subsequent time moments, the bell and step disturbances pass $\psi=0$ and the $\alpha(\psi)$ profile approaches the equilibrium solution where $\epsilon_{h, b}=2.5 \epsilon_{h, b}$, see 


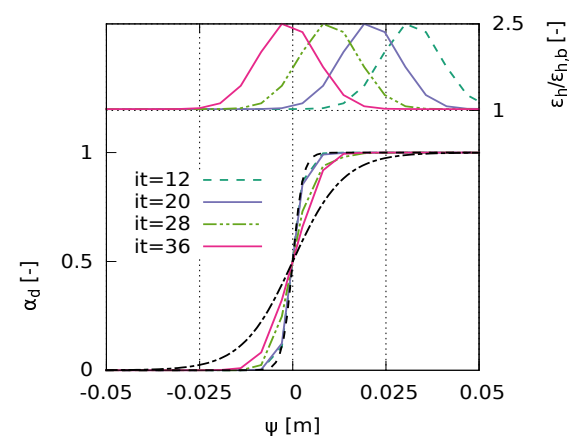

(a)

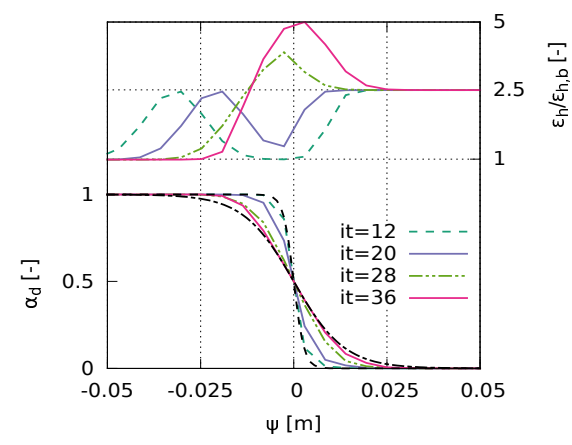

(c)

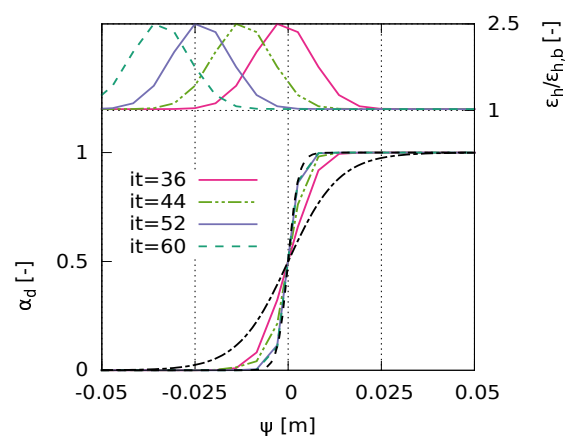

(b)

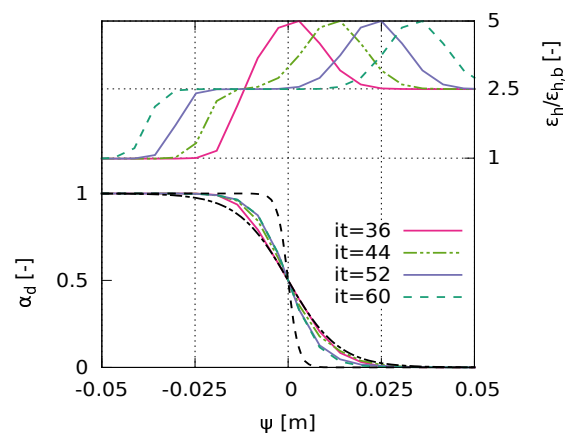

(d)

Figure 13: Comparison of $\alpha(\psi)$ profiles affected by the $\epsilon_{h}(\mathbf{x}, t)$ field (see Fig. 12) with the analytical solutions. Diagrams present the $\epsilon_{h}(\mathbf{x}, t)$ and $\alpha_{S, d}$ profiles drawn along the region $R 1$ (a),(b) and $R 2$ (c),(d) of the diagonal (see Fig. 12, it=12) at seven time moments it. Black lines mark the analytical profiles of $\alpha(\psi)$ where $\epsilon_{h}=\epsilon_{h, b}$ (dashed line) and $\epsilon_{h}=5 \epsilon_{h, b}$ (dashed-dotted line) obtained using Eq. (13).

Fig. $13 \mathrm{~d} i t>36$.

One notes that during the evolution in times $t, \tau$ the $\alpha(\psi)$ profile remains bounded between two extreme solutions obtained with $\epsilon_{h}=\epsilon_{h, b}$ (black dashed line) and $\epsilon_{h}=5 \epsilon_{h, b}$ (black dashed-dotted line), see Fig. 13. In the two- or threedimensional cases the integration (25) is carried out along the normal coordinate $\psi$ in the local system attached to the each point of regularized interface $\gamma$. Thus, as in the one-dimensional case, reduction to the equilibrium solution in the points where $\epsilon_{h}=$ const. is guaranteed by the design of the quadrature (31). 


\subsection{Two-dimensional semi-analytical solution with advection}

In this section, the semi-analytical approach described and verified in Sections $4.1-4.3$ is used to reconstruct the behavior of the intermittency region surrounding a two-dimensional circular drop with the radius $R=0.15[\mathrm{~m}]$ initially located at the point $(0.5,0.35)$ and advected in the divergence-free, constant, circular velocity field $\mathbf{u}=\left(u_{1}, u_{2}\right)=V_{0} / L(y-0.5,0.5-x)$ where $V_{0}=1[\mathrm{~m} / \mathrm{s}]$ and $L=1[m]$. The size of the computational domain, number of control volumes and settings of the solver of Eqs. (17) and (18) are the same as described in Section 4.3. In order to obtain the full rotation of the drop in the given velocity field $\mathbf{u}, \Delta t=2.5 \cdot 10^{-3}[s]$ and $N_{t}=2560$ time steps are required (the Courant number is $\sim 0.65$ ). The verification of the numerical methods and discretization of Eqs. (17) and (18) is described in detail in the previous works of the present author (Wacławczyk, 2015, 2017). Herein, in Appendix D the derivation of the Lagrangian scheme used to solve Eq. (17) in the known velocity field $\mathbf{u}$ is recalled.

The intermittency region surrounding the advected, circular interface is disturbed by the variable characteristic length scale field $\epsilon_{h}(\mathbf{x}, t)$ defined using Eq. (C.6) as the linear superposition of Eqs. (C.1) and (C.2). The results shown in Figs. $14-15$ present subsequent time moments it. $\Delta t$ (from top to bottom) in the history of the advected circular bubble the regularized interface of which is disturbed by the variable $\epsilon_{h}(\mathbf{x}, t)$ field. Each row in Figs. $14-15$ shows the same three iso-contours $\alpha\left(\psi=-4 \epsilon_{h, b}\right), \alpha(\psi=0), \alpha\left(\psi=4 \epsilon_{h, b}\right)$ set against $\alpha(\psi)$, $\epsilon_{h}(\mathbf{x}, t), \psi(\alpha)$ fields, from left to right respectively. The third column in Figs. $14-15$ presenting contours of the signed distance function $\psi(\alpha)$ is added to show the variation of the cumulative distribution $\alpha(\psi)$ is predicted in the region where $\mathbf{n}_{\gamma}$ is correctly defined, see Section 4.1 for the description of the numerical method.

One observes that the hat-like profile moving in the horizontal direction has a steep front and smooth tail, see Figs. $14-15$. It interferes with the bell shaped axis-symmetrical characteristic length scale variation resulting in the increase of its local values, the red color moving across the computational 


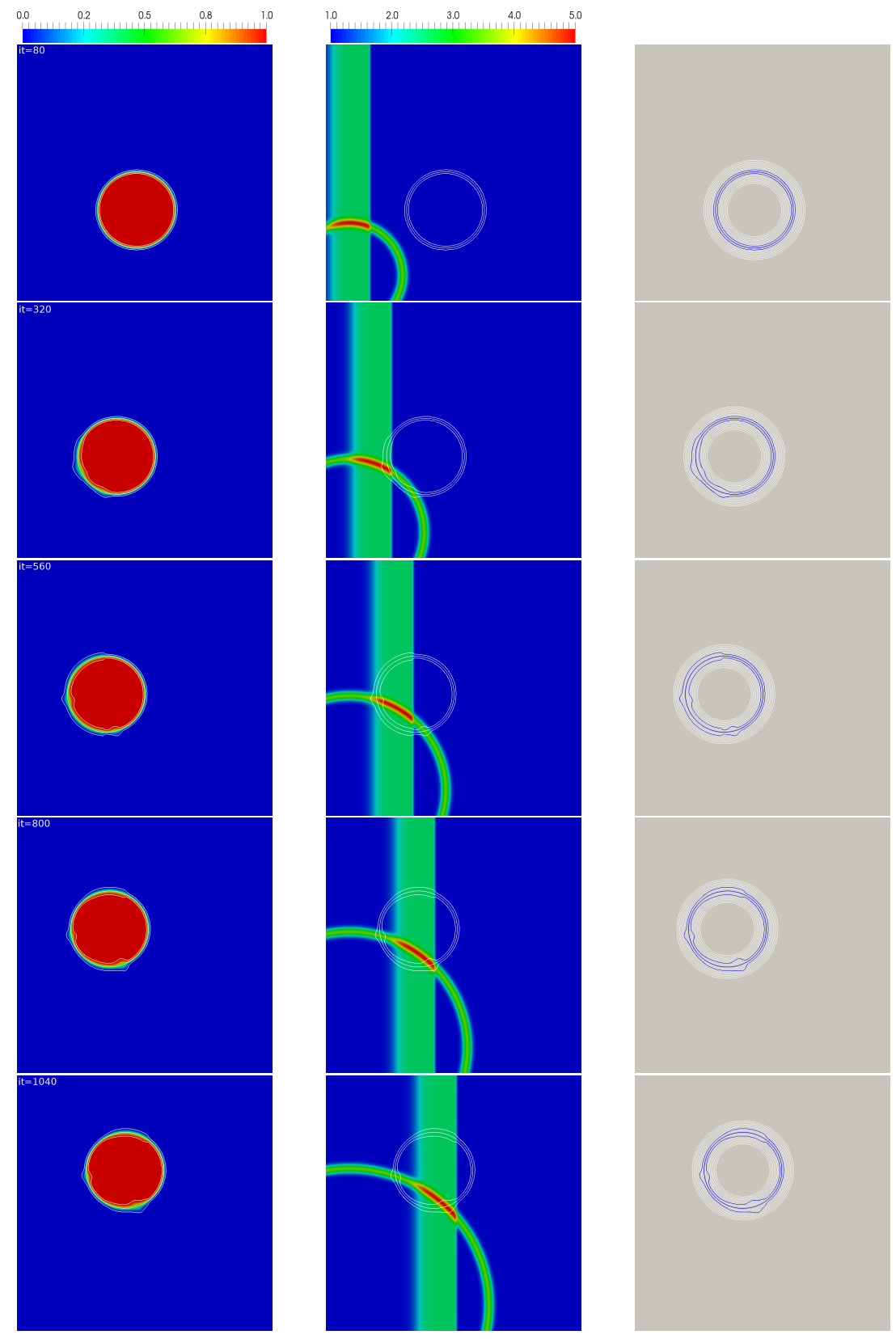

Figure 14: Evolution of the intermittency region affected by the variable field $\epsilon_{h}(\mathbf{x}, t)$. All figures show contours of $\alpha\left(\psi=-4 \epsilon_{h, b}\right), \alpha(\psi=0), \alpha\left(\psi=4 \epsilon_{h, b}\right)$, and from left to right fields of $0<\alpha(\psi)<1, \epsilon_{h, b} \leq \epsilon_{h}(\mathbf{x}, t)<5 \epsilon_{h, b}$, contours $-32 \epsilon_{h, b} \leq \psi(\alpha) \leq 32 \epsilon_{h, b}$. 

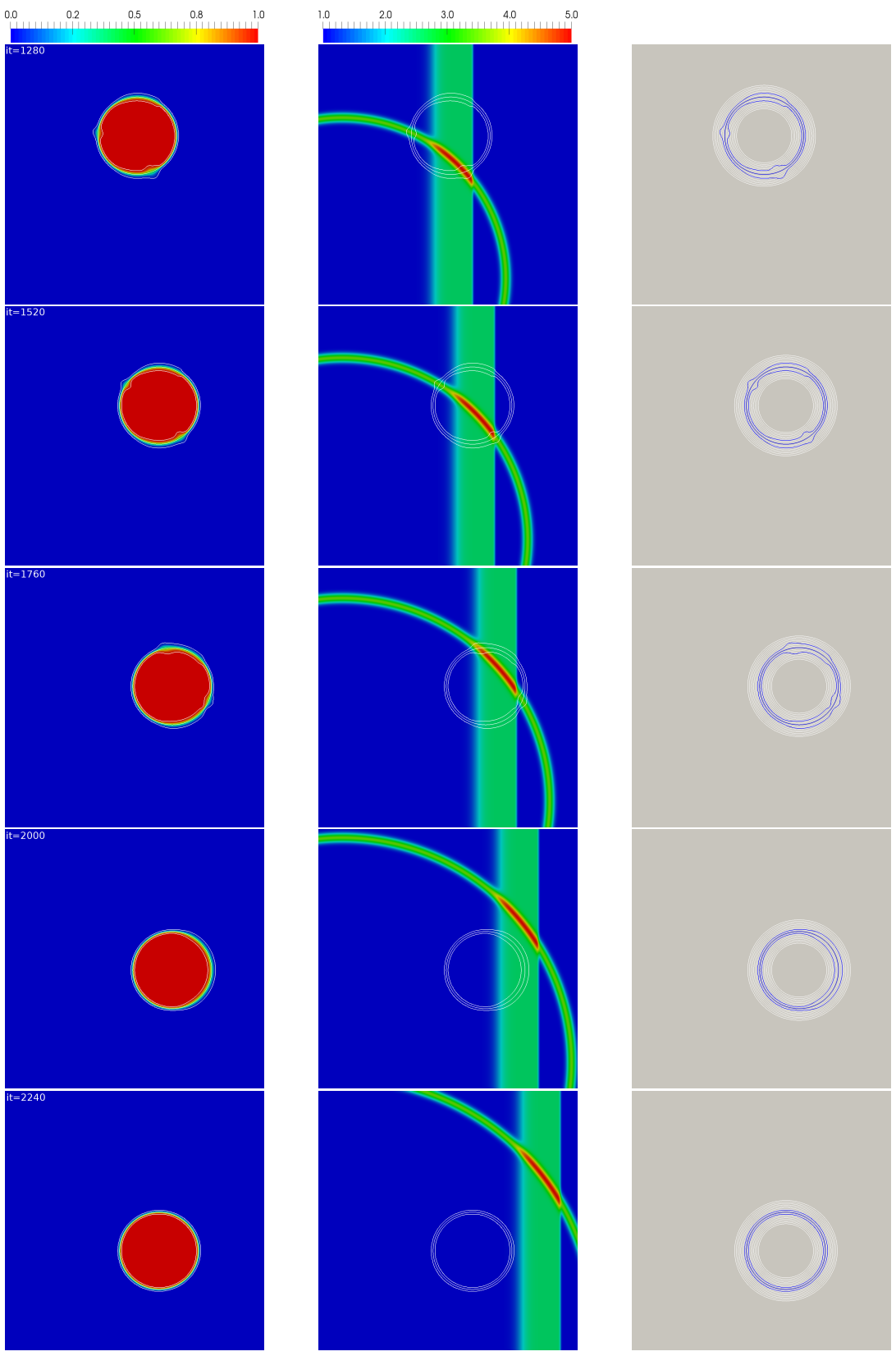

Figure 15: Evolution of the intermittency region affected by the variable field $\epsilon_{h}(\mathbf{x}, t)$. All figures show contours of $\alpha\left(\psi=-4 \epsilon_{h, b}\right), \alpha(\psi=0), \alpha\left(\psi=4 \epsilon_{h, b}\right)$, and from left to right fields of $0<\alpha(\psi)<1, \epsilon_{h, b} \leq \epsilon_{h}(\mathbf{x}, t)<5 \epsilon_{h, b}$, contours $-32 \epsilon_{h, b} \leq \psi(\alpha) \leq 32 \epsilon_{h, b}$. 
domain in the middle column of Figs. $14-15$; the $\epsilon_{h}(\mathbf{x}, t)$ field is bounded between $\epsilon_{h, b}<\epsilon_{h}(\mathbf{x}, t)<5 \epsilon_{h, b}$. We note the introduced numerical model is sensitive to the rapid changes in the magnitude of $\epsilon_{h}(\mathbf{x}, t)$. For example, in Fig. 14, it $=320,560$ one observes (along the circumference of axis-symmetrical variation) how the width of the intermittency region is affected by variable $\epsilon_{h}(\mathbf{x}, t)$. The proposed numerical method is sensitive to the local variations in the $\epsilon_{h}(\mathbf{x}, t)$ profile smoothness, too. In Fig. $15 i t=1280$, one notes (along the left-right borders of the step disturbance) how the steepness of the $\epsilon_{h}(\mathbf{x}, t)$ profile affects the $\alpha(\psi)$ field. As it can be observed in the last time moment presented in Fig. 15, in the domain where $\epsilon_{h}=\epsilon_{h, b}$ the intermittency region returns back to its original, equilibrium state. As it was mentioned previously this is achieved by the definition of quadrature (31) and integral in Eq. (29).

\section{Conclusions}

In the present paper, the non-equilibrium model of the intermittency region between two weakly miscible phases is introduced. This new multi-scale model is planned for the framework of one-fluid model of the (turbulent) two-phase flow. At first, the evolution equation of the intermittency region is derived from the stochastic viewpoint, see Eq. (12). Next, based on the mesoscopic and macroscopic interpretations of the intermittency region, conditions of its equilibrium and non-equilibrium state are explicated, see Section 2.1. The statistical interpretation of the solution to Eq. (12) is used to argue that the sharp interface tracked or captured in VOF, SLS models is localized inside the mesoscopic intermittency region (gas-liquid macroscopic interface) remaining in the equilibrium state, see Section 2.2. It is explained that the level-sets $H_{\gamma}=1 / 2, \psi=0$ are two-dimensional representations of the expected position of the mesoscopic interface $\Gamma$ disturbed by thermal fluctuations. This result unfolds how molecular effects are taken into account in VOF, SLS sharp interface models and answers how they reconstruct break up and coalescence (Perumanath et al., 2019).

The second part of the present paper refers to the case where the inter- 
mittency region could be in the non-equilibrium state as well. It is demonstrated that finding the stationary solution of Eq. (12) when the characteristic length scale $\epsilon_{h}(\mathbf{x}, t)$ characterizing the local thickness of the intermittency region $\epsilon_{h} \neq$ const. is equivalent to minimizing of the corresponding free energy functional, see Section 3.1. This result qualifies the stationary solution to Eq. (12) as the local equilibrium condition accounting for $\epsilon_{h}(\mathbf{x}, t)$. In Section 3.2, it is used to derive the modified mapping between the $\alpha(\psi)-\psi(\alpha)$ functions and the semi-analytical model of the evolving intermittency region, see Eq. (28). In Section 4 the new semi-numerical methods for the non-equilibrium solutions of Eq. (12) are introduced. In Sections (4.2-4.4) they are employed to reconstruct solutions of Eq. (12) with variable $\epsilon_{h}(\mathbf{x}, t)$ showing the complex behavior of the intermittency can be predicted with the approximate, semi-analytical model.

It is anticipated the semi-analytical approach introduced in the present paper could be used with the existing numerical sharp/diffusive interface models to approximate the effects of intermittency region non-equilibrium on the flow field. The only requirement is reconstruction of the signed distance function field based on the known expected position of the gas-liquid interface $\gamma$.

The modeling framework introduced in the present paper, is planned to be used in future statistical models of the macroscopic interface agitated by turbulent fields, or mesoscopic interface affected by variable thermal energy, pressure and/or concentration variations.

\section{Acknowledgments}

This work is supported by the grant of National Science Center, Poland (Narodowe Centrum Nauki, Polska) in the project "Statistical modeling of turbulent two-fluid flows with interfaces", ref. no. 2016/21/B/ST8/01010, ID:334165.

\section{Appendix A. Exact relations in surface averaging}

To derive Eq. (12) two exact relations between the ensemble $\langle\cdot\rangle$ and surface $\langle\cdot\rangle_{\Gamma} \Sigma$ averages are used. The first one is obtained directly from Eq. (3) as it 
implies

$$
\left\langle\mathbf{W}^{\prime} \cdot \nabla H_{\Gamma}\right\rangle=\left\langle\mathbf{W}^{\prime} \cdot \mathbf{n}_{\Gamma}\right\rangle_{\Gamma} \Sigma
$$

The second exact relation $\left\langle\mathbf{n}_{\Gamma}\right\rangle_{\Gamma} \Sigma=\nabla\left\langle H_{\Gamma}\right\rangle$ can be derived starting from the definition of the phase indicator function (Tryggvason et al., 2011)

$$
H_{\Gamma}(x, y, z, t)=\iiint_{V} \delta\left(x-x^{\prime}\right) \delta\left(y-y^{\prime}\right) \delta\left(z-z^{\prime}\right) d x^{\prime} d y^{\prime} d z^{\prime}
$$

and its gradient

$$
\nabla H_{\Gamma}(x, y, z, t)=-\iint_{\Gamma}\left(-\mathbf{n}_{\Gamma}^{\prime}\right) \delta\left(x-x^{\prime}\right) \delta\left(y-y^{\prime}\right) \delta\left(z-z^{\prime}\right) d \Gamma^{\prime} .
$$

In Eq. (A.3) relation $\nabla \delta\left(\mathbf{x}-\mathbf{x}^{\prime}\right)=-\nabla^{\prime} \delta\left(\mathbf{x}-\mathbf{x}^{\prime}\right)$ is used, moreover, it is noticed in the divergence theorem unit vector points outwards surface $\Gamma$ unlike the normal vector $\mathbf{n}_{\Gamma}=\nabla \Psi /|\nabla \Psi|$. According to (Pope, 1998), Eq. (A.3) can be rewritten as

$$
\nabla H_{\Gamma}(\mathbf{x}, t)=\iint_{\Gamma} \mathbf{n}_{\Gamma}(\mu, \lambda, t) \delta\left(\mathbf{x}-\mathbf{x}^{\prime}(\mu, \lambda, t)\right) A(\mu, \lambda, t) d \mu d \lambda
$$

where $\delta\left(\mathbf{x}-\mathbf{x}^{\prime}(\mu, \lambda, t)\right)\left[1 / m^{3}\right]$ is the three dimensional Dirac's delta function. Therefore, in the local orthonormal coordinate system $\mu, \lambda, \Psi$ of infinitesimally small surface element $d \Gamma^{\prime}=A(\mu, \lambda, t) d \mu d \lambda$, where $\Psi$ is the coordinate in the normal direction, Eq. (A.4) reads

$$
\nabla H_{\Gamma}(\Psi)=\iint_{\Gamma} \mathbf{n}_{\Gamma} \delta(\mu) \delta(\lambda) \delta\left(\Psi-\Psi^{\prime}(\mu, \lambda, t)\right) A(\mu, \lambda, t) d \mu d \lambda=\delta_{\Gamma}(\Psi) \mathbf{n}_{\Gamma}
$$

in addition, Eq. (A.5) let us show

$$
\frac{\partial H_{\Gamma}(\Psi)}{\partial \Psi}=\delta_{\Gamma}(\Psi)
$$

Using the surface average definition given by Eq. (4), the surface average of the normal vector $\mathbf{n}_{\Gamma}$ is obtained as

$$
\left\langle\mathbf{n}_{\Gamma}\right\rangle_{\Gamma} \Sigma=\iint_{\Gamma}\left\langle\mathbf{n}_{\Gamma} \delta(\mu) \delta(\lambda) \delta_{\Gamma}\left(\Psi-\Psi^{\prime}(\mu, \lambda, t)\right) A(\mu, \lambda, t)\right\rangle d \mu d \lambda
$$

where $\Sigma$ is defined by Eq. (6). Finally, taking the ensemble average $\langle\cdot\rangle$ of Eq. (A.5) and comparing with Eq. (A.7) results in the second exact relation

$$
\nabla\left\langle H_{\Gamma}\right\rangle=\left\langle\mathbf{n}_{\Gamma}\right\rangle_{\Gamma} \Sigma
$$




\section{Appendix B. Calculation of the free energy functional derivative}

In order to compute the functional derivative of Eq. (19) with $\epsilon_{h}(\mathbf{x}, t)$ we use the following definition. Given a functional

$$
G[\alpha]=\int_{V} g(\psi, \alpha(\psi), \nabla \alpha(\psi)) d V,
$$

its functional derivative is obtained as

$$
\frac{\delta G}{\delta \alpha}=\frac{\partial g}{\partial \alpha}-\nabla \cdot\left[\frac{\partial g}{\partial \nabla \alpha}\right] .
$$

The first term in Eq. (B.2), where $G=F$ and $F$ is given by Eq. (19), results in

$$
\frac{\partial f}{\partial \alpha}=\sigma\left[\frac{2 \alpha(1-\alpha)}{\epsilon_{h}}(1-2 \alpha)+\frac{\delta k}{\delta \alpha}\right] .
$$

Since $\delta k / \delta \alpha$ is given by Eq. (20) and it does not depend explicitly on $\epsilon_{h}(\mathbf{x}, t)$, Eq. (B.3) reads

$$
\frac{\partial f}{\partial \alpha}=2 \sigma\left[\frac{\alpha(1-\alpha)}{\epsilon_{h}}(1-2 \alpha)+\alpha(1-\alpha) \nabla \cdot \mathbf{n}_{\gamma}\right] .
$$

The second term in Eq. (B.2), where $G=F$ and $F$ is given by Eq. (19), equals

$$
\nabla \cdot\left[\frac{\partial f}{\partial \nabla \alpha}\right]=2 \sigma\left(\epsilon_{h} \nabla^{2} \alpha+\nabla \epsilon_{h} \cdot \nabla \alpha\right) .
$$

Therefore, to minimize the functional $F[\alpha]$, we search for

$$
\frac{\delta F}{\delta \alpha}=\epsilon_{h} \nabla^{2} \alpha+\nabla \epsilon_{h} \cdot \nabla \alpha-\frac{\alpha(1-\alpha)}{\epsilon_{h}}\left[(1-2 \alpha)+\epsilon_{h} \nabla \cdot \mathbf{n}_{\gamma}\right]=0 .
$$

\section{Appendix C. Variations of the characteristic length scale field}

In the present work it is assumed variations of the characteristic length scale of the regularized interface $\epsilon_{h}(\mathbf{x}, t)$ are known and given by the predefined formulas. The step profile

$$
\epsilon_{h, S}(\mathbf{x}, t)=\epsilon_{h, b}\left[1+\frac{H_{S}}{1+\exp \left(-\frac{f_{S}(\mathbf{x}, t)}{W_{S} \epsilon_{h, b}}\right)}\right]
$$

or the bell shaped profile

$$
\epsilon_{h, B}(\mathbf{x}, t)=\epsilon_{h, b}\left[1+H_{B} \exp \left(-\frac{f_{B}(\mathbf{x}, t)}{W_{B} \epsilon_{h, b}}\right)^{2}\right] .
$$


In the one-dimensional cases presented in Figs. $4-5, H_{S}=H_{B}=W_{S}=1, W_{B}=6$ and $\epsilon_{h, b}=\Delta x$. The functions $f_{S}, f_{B}$ in Eqs. (C.1) and (C.2) are both set to $f(x, t)=x-x_{\Gamma}+\epsilon_{h, b}(36-i t)$ where $x_{\Gamma}=0.5$ is position of the interface and $i t=1, \ldots, 72$ denotes the physical time iteration number.

In the two dimensional case studied in Section 4.3, the variation of $\epsilon_{h}(\mathbf{x}, t)$ presented in Figs. $12-13$ is obtained as

$$
\epsilon_{h}(\mathbf{x}, t)=\frac{1}{2}\left[\epsilon_{h, S}(\mathbf{x}, t)+\epsilon_{h, B}(\mathbf{x}, t)\right]
$$

where in Eq. (C.2)

$$
f_{B}(\mathbf{x}, t)=R-R_{D}+\epsilon_{h, b}(72-i t)
$$

and $\epsilon_{h, b}=\sqrt{2} \Delta x / 4, R=\sqrt{(\mathbf{x}-\mathbf{r})^{2}}, \mathbf{r}=(0.5,0.5)$ determines the center and $R_{D}=0.2$ sets initial radius of axis-symmetrical part of $\epsilon_{h}(\mathbf{x}, t)$. In Eq. (C.3) the step, oblique variation is governed by

$$
f_{S}(\mathbf{x}, t)=-(x-0.62)-(y-0.62)-\epsilon_{h, b} \cdot i t
$$

moreover $H_{B}=H_{S}=4, W_{B}=8, W_{S}=2, i t=1, \ldots, 16$.

In Section 4.4 where the semi-analytical solution with advection is studied, the following superposition of $\epsilon_{h, B}$ and $\epsilon_{h, S}$ is employed

$$
\epsilon_{h}(\mathbf{x}, t)=\frac{1}{2}\left[\epsilon_{h, b}+\epsilon_{h, S 1}(\mathbf{x}, t)-\epsilon_{h, S 2}(\mathbf{x}, t)+\epsilon_{h, B}(\mathbf{x}, t)\right]
$$

where $\epsilon_{h, b}=\sqrt{2} \Delta x / 4$, variation of $\epsilon_{h, B}$ with $H_{B}=5, W_{B}=12$, is carried out using the function $f_{B}(t)$ given by Eq. (C.4) where $R_{D}=0.2, \mathbf{r}=(0.1,0.1)$. Step profiles in Eq. (C.6) are defined using $H_{S 1}=H_{S 2}=3, W_{S 1}=1$ and $W_{S 2}=10$, moreover, they are driven in time by the functions $f_{S 1}(x, t)=x-0.15-0.25 \epsilon_{h, b} \cdot$ it and $f_{S 2}(x, t)=f_{S 1}(x, t)+0.15$, respectively.

\section{Appendix D. Derivation of the Lagrangian scheme, constrained in- terpolation}

The rearrangement of terms in the advection equation (17) leads to

$$
\frac{1}{\alpha(1-\alpha)} \frac{\partial \alpha}{\partial t}=-\frac{1}{\epsilon_{h}}|\nabla \psi| \mathbf{w} \cdot \mathbf{n}_{\gamma} .
$$


The left hand side is now integrated between $\alpha^{n}$ and $\alpha^{n+1}$, whereas the right hand side between $t^{n}$ and $t^{n+1}$ resulting in

$$
\left.\ln \left(\frac{\alpha}{1-\alpha}\right)\right|_{\alpha^{n}} ^{\alpha^{n+1}}=-\int_{t^{n}}^{t^{n+1}} \frac{1}{\epsilon_{h}}|\nabla \psi| \mathbf{w} \cdot \mathbf{n}_{\gamma} d t,
$$

where $n, n+1$ denotes old and new time levels, respectively. Integration given by Eq. (D.2) derives the following scheme for advancement of $\alpha(\psi)-\psi(\alpha)$ in time $t$, given by the formula

$$
\alpha^{n+1}=\frac{\alpha^{n} \exp \left[I\left(t^{n}\right)\right]}{1-\alpha^{n}\left(1-\exp \left[I\left(t^{n}\right)\right]\right)},
$$

where the RHS integral in Eq. (D.2) is denoted as $I\left(t^{n}\right)$. This integral must be approximated by the appropriate quadrature; in the present work we adopt the second-order Adams-Bashfort method leading to

$$
I\left(t^{n}\right) \approx-\left[\frac{3}{2} f\left(t^{n}, \psi^{n}\right)-\frac{1}{2} f\left(t^{n-1}, \psi^{n-1}\right)\right] \Delta t,
$$

where $f=|\nabla \psi| \mathbf{n}_{\gamma} \cdot \mathbf{w} / \epsilon_{h}$. The semi-analytical, explicit scheme given by Eqs. (D.3) and (D.4) is second-order accurate in time and no spatial discretization of $\alpha(\psi)$ is needed. It is noted that in the present work $\epsilon_{h}^{n+1}=\epsilon_{h}^{n}$ as the advection equation (17) is always solved with $\epsilon_{h, b}=$ const. in the semi-analytical case.

During numerical solution of Eq. (18) in time $\tau$, to obtain $\alpha_{d c}$ shown in Figs. $4-5$ and Fig. 9, the constrained interpolation (Wacławczyk, 2017) is used to determine $\tilde{\delta}(\alpha)=\alpha(1-\alpha)$. The constrained interpolation in the present work is summarized below

$$
\begin{aligned}
\psi_{f} & \approx \frac{1}{2}\left(\psi_{P}+\psi_{F}\right)+\mathcal{O}\left(\Delta x^{2}\right), \\
\alpha_{f} & =\alpha\left(\psi_{f}\right)=\frac{1}{1+\exp \left(-\psi_{f} I(\psi)\right)},
\end{aligned}
$$

where subscripts $F, f, P$ denote the neighbor control volume $F$ and face $f$ of the given control volume $P$, respectively. $I(\psi)$ is the quadrature defined by Eq. (30) or Eq. (31). 


\section{Appendix E. Error norms}

To show convergence during the numerical solution of Eqs. (18) and (27) where $\alpha(\psi)$ is disturbed by $\epsilon_{h, S}(\mathbf{x}, t)$ or $\epsilon_{h, B}(\mathbf{x}, t)$ (see Eqs. (C.2) and (C.1), respectively), in Figs. $6,7,8$ the $L_{1, \tau}$ error norm is plotted after each physical time iteration $i t$. This first-order norm is defined as follows

$$
L_{1, \tau}=\frac{1}{N_{c}} \sum_{i=1}^{N_{c}}\left|\alpha_{i}^{n+1}-\alpha_{i}^{n}\right|,
$$

where $N_{c}$ is the number of control volumes and $n+1$ denotes a new level of the pseudo-time $\tau$.

In Fig. 10 the norms $L_{1, S}(\alpha)$ and $L_{1, B}(\alpha)$ are used. They are employed to access the spatial error of the integration procedure introduced in Section 4.1 during coupled (dc) and semi-analytical (d) solutions. Their definition uses the analytical profiles $\alpha_{c n v}(\mathbf{x}, T)$, representing known, equilibrium solutions of Eqs. (18) and (27) after predefined physical time $T=i t \cdot \Delta t$ where herein $i t=72$, $\Delta t=10^{-3}[s] . \quad \alpha_{c n v}(\mathbf{x}, T)$ is defined by Eq. (13) with $\epsilon_{h}=2 \epsilon_{h, b}$ or $\epsilon_{h}=\epsilon_{h, b}$ in the case when $\epsilon_{h}=\epsilon_{h, S}$ or $\epsilon_{h}=\epsilon_{h, B}$, respectively. If discretized analog of $\alpha_{c n v}(\mathbf{x}, T)$ is given by $\alpha_{i, c n v}$ the norm $L_{1, D}(\alpha)$, where $D=S$ or $B$ is defined at each time iteration $i t$, as

$$
L_{1, D}^{i t}=\frac{1}{N_{c}} \sum_{i=1}^{N_{c}}\left|\alpha_{i}^{i t}-\alpha_{i, c n v}\right| .
$$

\section{References}

Aarts, D. G. A. L., Schmidt, M., Lekkerkerker, H. N. W., 2004. Direct visual observation of thermal capillary waves. Science, 304, 847-850. doi:10.1126/ science.1097116.

Allen, S., Cahn, J., 1979. A microscopic theory for antiphase domain boundary motion and its application to antiphase domain coarsening. Acta Metall., 27, 1085-1095. 
Anderson, D. M., McFadden, G. B., Wheeler, A. A., 1998. Diffuse-Interface Methods in Fluid Mechanics. Annu. Rev. Fluid Mech., 30, 139-165. doi:10. 1146/annurev.fluid.30.1.139.

Aniszewski, W., Bogusławski, A., Marek, M., Tyliszczak, A., 2012. A new approach to sub-grid surface tension for les of two-phase flows. Journal of Computational Physics, 231, 7368 - 7397. URL: http: //www.sciencedirect.com/science/article/pii/S0021999112003890. doi:https://doi.org/10.1016/j.jcp.2012.07.016.

Brocchini, M., Peregrine, D. H., 2001a. The dynamics of strong turbulence at free surfaces. Part 1. Description. J. Fluid Mech., 449, 225-254.

Brocchini, M., Peregrine, D. H., 2001b. The dynamics of strong turbulence at free surfaces. Part 2. Free-surface boundary conditions. J. Fluid Mech., 449, $255-290$.

Cahn, J. W., Hilliard, J. E., 1958. Free Energy of a Nonuniform System. I. Interfacial Free Energy. J. Chem. Phys., 28, 258-267. doi:http://dx.doi. org/10.1063/1.1744102.

Chiu, P.-H., Lin, Y.-T., 2011. A conservative phase field method for solving incompressible two-phase flows. J. Comp. Phys., 230, 185-204. doi:http: //dx.doi.org/10.1016/j.jcp.2010.09.021.

Deike, L., Melville, W. K., Popinet, S., 2016. Air entrainment and bubble statistics in breaking waves. Journal of Fluid Mechanics, 801, 91129. doi:10. 1017/jfm.2016.372.

Elghobashi, S., 2019. Direct numerical simulation of turbulent flows laden with droplets or bubbles. Annual Review of Fluid Mechanics, 51, 217-244. URL: https://doi.org/10.1146/annurev-fluid-010518-040401. doi:10.1146/ annurev-fluid-010518-040401. arXiv:https://doi.org/10.1146/annurev-fluid-010518-040401.

Faust, J. A., 2018. Foreword. In J. A. Faust, and J. E. House (Eds.), Physical Chemistry of Gas-Liquid Interfaces Developments in Physical 
\& Theoretical Chemistry (pp. Foreword, xvii). Elsevier. URL: http:// Www.sciencedirect.com/science/article/pii/B9780128136416120011. doi:https://doi .org/10.1016/B978-0-12-813641-6.12001-1.

Fedeli, L., 2017. Computer simulations of phase field drops on superhydrophobic surfaces. J. Comp. Phys., 344, 247-259. doi:http://dx.doi. org $/ 10.1016 / j \cdot j c p .2017 .04 .068$.

Gibbs, J. W., 1874. On the equilibrium of heterogeneous substances. Academy. Gruszczyński, G., Mitchell, T., Leonardi, C., Łaniewski-Wołłk, Ł., Barber, T., 2020. A cascaded phase-field lattice boltzmann model for the simulation of incompressible, immiscible fluids with high density contrast. Computers $\&$ Mathematics with Applications, 79, 1049-1071. URL: http: //www.sciencedirect.com/science/article/pii/S0898122119304158. doi:https ://doi.org/10.1016/j. camwa.2019.08.018.

Guo, X., Shen, L., 2010. Interaction of a deformable free surface with statistically steady homogeneous turbulence. J. Fluid Mech., 658, 32-62.

Herrmann, M., 2013. A sub-grid surface dynamics model for sub-filter surface tension induced interface dynamics. Computers \& Fluids, 87, 92 - 101. URL: http://www.sciencedirect.com/science/article/pii/ S0045793013000637. doi:https://doi.org/10.1016/j.compfluid.2013. 02.008. USNCCM Moving Boundaries.

Höhne, T., Vallée, C., 2009. Modelling of stratified two phase flows using an interfacial area density model. In Computational Methods in Multiphase Flow $V$ (pp. 123-133). New Forest, United Kingdom.

Hong, W.-L., Walker, D., 2000. Reynolds-averaged equations for free surface flows with application to high-Froude-number jet spreding. J. Fluid Mech., 417, 183-209.

Kajzer, A., Pozorski, J., 2020. A weakly compressible, diffuse-interface model for two-phase flows. Flow, Turbulence and Combustion, . URL: http: 
//www.sciencedirect.com/science/article/pii/S0301932219304732.

doi:https://doi.org/10.1007/s10494-020-00164-8.

Komrakova, A. E., Eskin, D., Derksen, J. J., 2015. Numerical study of turbulent liquid-liquid dispersions. AIChE Journal, 61, 2618-2633. URL: https://aiche.onlinelibrary. wiley.com/doi/abs/10.1002/aic.14821. doi:10.1002/aic.14821. arXiv:https://aiche.onlinelibrary.wiley.com/doi/pdf/10.1002/aic.14821.

Labourasse, E., Lacanette, D., Toutant, A., Lubin, P., Vicent, S., Lebaigue, O., Caltagirone, J.-P., Sagaut, P., 2007. Towards large eddy simulation of isothermal two-phase flows: Governing equations and a priori tests. Int. J. Multiphase Flow, 33, 1-39.

Lu, J., Tryggvason, G., 2018. Direct numerical simulations of multifluid flows in a vertical channel undergoing topology changes. Phys. Rev. Fluids, 3, 084401. URL: https://link.aps.org/doi/10.1103/PhysRevFluids.3. 084401. doi:10.1103/PhysRevFluids.3.084401.

McCaslin, J. O., Desjardins, O., 2014. A localized re-initialization equation for the conservative level set method. J. Comp. Phys., 262, 408-426. doi:http: //dx.doi.org/10.1016/j.jcp.2014.01.017.

Mirjalili, S., Ivey, C. B., Mani, A., 2020. A conservative diffuse interface method for two-phase flows with provable boundedness properties. Journal of Computational Physics, 401, 109006. URL: http: //www.sciencedirect.com/science/article/pii/S0021999119307119. doi:https://doi.org/10.1016/j.jcp.2019.109006.

Olsson, E., Kreiss, G., 2005. A conservative level-set method for two phase flow. J. Comp. Phys., 210, 225-246.

Osher, S., Fedkiw, R., 2003. Level Set Methods and Dynamic Implicit Surfaces. Springer Verlag, INC. New-York. 
Osher, S., Sethian, J. A., 1988. Fronts propagating with curvature-dependent speed: Algorithms based on Hamilton-Jacobi formulations. J. Comp. Phys., 79, 12 - 49. doi:http://dx.doi.org/10.1016/0021-9991 (88)90002-2.

Perumanath, S., Borg, M. K., Chubynsky, M. V., Sprittles, J. E., Reese, J. M., 2019. Droplet coalescence is initiated by thermal motion. Phys. Rev. Lett., 122, 104501. URL: https://link.aps.org/doi/10.1103/PhysRevLett. 122.104501. doi:10.1103/PhysRevLett.122.104501.

Pope, S., 1998. The evolution of surfaces in turbulence. Int. J. Eng. Sciences, $26,445-469$.

Prosperetti, A., Tryggvason, G., 2007. Computational Methods for Multiphase Flow. Cambridge University Press.

Saeedipour, M., Schneiderbauer, S., 2019. A new approach to include surface tension in the subgrid eddy viscosity for the two-phase les. International Journal of Multiphase Flow, 121, 103128. URL: http: //www. sciencedirect.com/science/article/pii/S0301932219304732. doi:https ://doi.org/10.1016/j.ijmultiphaseflow . 2019.103128.

Skartlien, R., Hu, B., Palmer, T., Staff, G., Sollum, E., 2014. A statistical model for the average volume fraction profile through the mixing zone in turbulent stratified gasliquid flow. International Journal of Multiphase Flow, 59, 160 - 172. URL: http: //www.sciencedirect.com/science/article/pii/S0301932213001870. doi:https://doi.org/10.1016/j.ijmultiphaseflow. 2013.11.002.

Smolentsev, S., Miraghaie, R., 2005. Study of a free surface in open-channel water flows in the regime from "weak" to "strong" turbulence. Int. J. Multiphase Flows, 31, 921-939.

Soligo, G., Roccon, A., Soldati, A., 2019. Breakage, coalescence and size distribution of surfactant-laden droplets in turbulent flow. Journal of Fluid Mechanics, 881, 244282. doi:10.1017/jfm.2019.772. 
Sussman, M., Smith, K., Hussaini, M., Ohta, M., Zhi-Wei, R., 2007. A sharp interface method for incompressible two-phase flows. Journal of Computational Physics, 221, 469-505.

Thiesset, F., Duret, B., Mnard, T., Dumouchel, C., Reveillon, J., Demoulin, F. X., 2020. Liquid transport in scale space. Journal of Fluid Mechanics, 886, A4. doi:10.1017/jfm.2019.1056.

Toutant, A., Fournier, C., Chandesris, M., Jamet, D., Lebaigue, O., 2007. Interfacial conditions at a filtered interface for LES two-phase flows. In Proceedings of the 6-th International Conference on Multiphase Flow. Leipzig, Germany.

Tryggvason, G., Scardovelli, R., Zaleski, S., 2011. Direct Numerical Simulations of Gas-Liquid Multiphase Flows. Cambridge University Press.

Vrij, A., 1973. Light scattering from liquid interfaces. Chemie Ingenieur Technik, 45, 1113-1114. doi:10.1002/cite.330451807.

van der Waals, J., D., 1893. Thermodynamische theorie der capillariteit in de onderstelling van continue dichtheidsveranderiing. Verhand. Kon. Akad. V Wetensch. Amst., 20. Dutch; English Translation in J. Stat. Phys., 1979, 20, 197.

Wacławczyk, M., Oberlack, M., 2011. Closure proposals for the tracking of turbulence-agitated gas-liquid interfaces in stratified flows. Int. J. Multiphase Flow, 37, 967-976.

Wacławczyk, M., Wacławczyk, T., 2015. A priori study for the modelling of velocity-interface correlations in the stratified air-water flows. Int. J. Heat Fluid Flow, 52, 40 - 49. doi:http://dx.doi.org/10.1016/j. ijheatfluidflow.2014.11.004.

Wacławczyk, T., 2015. A consistent solution of the re-initialization equation in the conservative level-set method. J. Comp. Phys., 299, 487-525. doi:http: //dx.doi.org/10.1016/j.jcp.2015.06.029. 
Wacławczyk, T., 2017. On a relation between the volume of fluid, level-set and phase field interface models. International Journal of Multiphase Flow, 97, 60 - 77. URL: http://www.sciencedirect.com/science/ article/pii/S0301932216307856. doi:https://doi.org/10.1016/j. ijmultiphaseflow.2017.08.003.

Wacławczyk, T., Wacławczyk, M., Kraheberger, S. V., 2014. Modeling of turbulence-interface interactions in stratified two-phase flows. Journal of Physics: Conference Series, 530. 